\title{
Exchangeable Random Networks
}

\author{
F. Bassetti, M. Cosentino Lagomarsino, and S. Mandrà
}

Abstract. We introduce and study a class of exchangeable random graph ensembles. They can be used as statistical null models for empirical networks, and as a tool for theoretical investigations. We provide general theorems that characterize the degree distribution of the ensemble graphs, together with some features that are important for applications, such as subgraph distributions and kernel of the adjacency matrix. A particular case of directed networks with power-law out-degree is studied in more detail, as an example of the flexibility of the model in applications.

\section{Introduction}

Random graphs have attracted much interest as null and positive models for many real-world systems involving many interacting agents, such as the Internet, epidemics, and social and biological interactions (see, for instance, [Strogatz 01, Newman 03a, Newman et al. 06, Montoya et al. 06]). In many of these instances, one is naturally confronted with properties that differ from the classical Erdős-Rényi model. We recall that, in the Erdős-Rényi model, edges in the graph exist independently from each other, with a fixed probability (dependent on the dimension of the graph). While for the Erdős-Rényi model analytical expressions for many of the relevant observable properties of the graph (such as the diameter, clustering coefficient, component size distributions, subgraph distribution, giant component, etc.) are available, less is known for other kinds of models. In recent

(C) A K Peters, Ltd.

I542-795I/07 \$0.50 per page 
years, in connection with the availability of large-scale data on real-life networks, many studies addressing random graph models going beyond the Erdős-Rényi model have appeared. Two studies that are worth mentioning are the so-called "small-world" model [Watts and Strogatz 98] and the preferential-attachment model [Barabasi and Albert 99], addressing the empirically observable phenomena of short shortest-paths and power-law degree distributions, respectively. This new wave of models has affected also the mathematical literature (see, for instance, [Aiello et al. 01, Aiello et al. 02, Bollobás and Riordan 03b, Bollobás and Riordan 03a, Bollobás et al. 03, Bollobás and Riordan 04a, Bollobás and Riordan 04b, Chung and Lu 03, Chung and Lu 04, Chung and Lu 06b, Newman 03a, Newman 03b]). Among the many recent mathematical books on the subject, we would like to mention, for classical random graph theory, the works by Kolchin [Kolchin 99] and by Bollobás [Bollobás 01] and, for more recent models of random graphs, the books by Chung and Lu [Chung and Lu 06a] and by Durrett [Durrett 07]. From a statistical point of view, which we adopt here, it is natural to seek a parameterizable stochastic model of complex graphs that would be at the same time flexible for practical use and mathematically tractable for theoretical exploration. Moreover, it is desirable that the qualitative properties of the model should emerge from some simple unifying mathematical structure rather than from ad-hoc considerations (see [Aiello et al. 02, Bollobás 01, Bollobás and Riordan 03a, Bollobás et al. 03, Bollobás and Riordan 04a, Chung and Lu 04, Newman et al. 06]).

The aim of this paper is to present a general class of random graphs that addresses these needs. It was introduced by Bassetti et al. in a particular case [Bassetti et al. 07], connected to the study of null models for transcriptional regulation networks [Babu et al. 04]. The defining property of the graph ensemble is the exchangeable structure of its degree correlations. This symmetry property makes it particularly apt to be used as a statistical null model. The most important advantages of such an approach are the following: (i) Much as in the Erdős-Rényi model, some observables can be easily computed analytically for finite sizes and asymptotically, rather than estimated numerically. (ii) It is fast and versatile in computational implementations and statistical applications. As we will show in the different sections of this paper, many observables that are commonly useful in the analysis of large-scale networks are particularly simple to access with our ensemble. In order to show the range of applicability, we discuss multiple applications to observables in the model graphs rather than presenting a very detailed analysis on a single graph feature. In the use as a null model, differently from other approaches used in the study of transcriptional and other networks [Itzkovitz et al. 03, Rao et al. 96, Chen et al. 05], our generating method for random graphs is not de- 
signed to conserve the degree sequence of the observed real graph, but rather as a method to generate graphs with degree distributions having certain prescribed properties.

The paper is structured as follows. Section 2 introduces a rather general class of random directed network ensembles that can be produced with the same defining principle of exchangeability, and discusses some simple variants. The following part is intended to show how the structure of the proposed model is useful in the the study of many relevant topological features of the ensemble. To this aim, in Section 3 we prove some theorems that characterize the degree distributions and the distribution of the size of the "hub" (or the maximally connected node). In particular, we show that the model can generate an ensemble characterized by a Poisson limit distribution for the in-degree, and a mixture of Poisson limit distributions for the out-degree. This important property enables to obtain a limit out-degree distribution with power-law tails. In the same section, we show that the probability that the graph is disconnected goes to 1 as the size of the graph diverges. Section 4 gives some results concerning the mean number of subgraphs (a quantity of some importance in many applications), roots, and leaves. Section 5 considers a particular Boolean optimization problem defined on the graph, which emerges in statistical physics and theoretical computer science. More precisely, we will give some results concerning the nontrivial problem of the dimension of the kernel of the adjacency matrix. In Section 6 we briefly comment the two variants of the main model. Finally, Section 7 contains the detailed analysis of a simple two-parameter ensemble derived from the general model presented in Section 2. Some of the proofs are deferred to Section 8.

\section{The Model}

Although the ideas we describe are applicable to both directed and undirected graphs, we will mainly consider here the case of directed graphs. Any directed random graph $G_{n}$ with $n$ nodes is completely specified by its adjacency matrix $\mathbb{X}_{n}=\mathbb{X}\left(G_{n}\right)=\left[X_{i, j}^{(n)}\right]_{i, j=1, \ldots, n}$, where $X_{i, j}^{(n)}=1$ if there is a directed edge $i \rightarrow j$, and 0 otherwise. In many applications, such as transcription networks instead of square matrices, one may also consider rectangular matrices. The reason for this is that in some situations it is reasonable to assume that, while all nodes can receive edges, only a fraction of nodes can send them out (see [Bassetti et al. 07] for an introduction to this problem). Hence, in what follows we will deal with rectangular matrices $m_{n} \times n$. As we will see in Section 7 , this is a necessary choice for networks with power-law degree distributions having exponent equal to or less than 2 (thus with diverging average) to obtain nontrivial asymptotics. 
One of the interests of our procedure is the fact that it can produce graphs with different in- and out-degree distributions. Naturally, if the graph is generated by throwing independently each directed edge with a fixed probability - as in the case of (undirected) Erdös-Rényi graphs - this is not possible. In order to build a random graph with different in- and out-degree distributions, one must give up total independence and allow some kind of dependence among edges. In particular, maintaining the maximal symmetry leads to the choice of exchangeability.

\section{I. Partially Exchangeable Random Graphs}

The first general class we will consider includes directed graphs whose in- or out-degrees, i.e., the columns or the rows of $\mathbb{X}_{n}$, respectively, are exchangeable, while the out- or in-degrees are stochastically independent. Differently put, our model ensemble can be defined using the following generative algorithm. For each row of $\mathbb{X}_{n}$, independently, (i) throw a bias $\theta$ from a prescribed probability distribution $\pi_{n}$ on $[0,1]$, and (ii) set the row elements of $\mathbb{X}_{n}$ to be 0 or 1 according to the toss of a coin with bias $\theta$. Since each row is thrown independently, the resulting probability law is

$$
\begin{aligned}
P\left\{X_{i, j}^{(n)}\right. & \left.=e_{i, j}, i=1, \ldots, m_{n}, j=1, \ldots, n\right\} \\
& =\prod_{i=1}^{m_{n}} \int_{[0,1]} \theta_{i}^{\sum_{j=1}^{n} e_{i, j}}\left(1-\theta_{i}\right)^{n-\sum_{j=1}^{n} e_{i, j}} \pi_{n}\left(d \theta_{i}\right),
\end{aligned}
$$

where $e_{i, j} \in\{0,1\}, i, j=1, \ldots n$. In other words, each row of $\mathbb{X}\left(G_{n}\right)$ is independent from the others with exchangeable law directed by $\pi_{n}$. One can apply an identical procedure to the transposed matrix of $\mathbb{X}_{n}$ and switch the role of inand out-degrees.

It is worth recalling that a random vector, say $\left(Y_{1}, \ldots, Y_{n}\right)$, is said to be exchangeable if its law is invariant under any permutation, that is, if for any permutation $\sigma$ of $\{1, \ldots, n\},\left(Y_{1}, \ldots, Y_{n}\right)$ and $\left(Y_{\sigma(1)}, \ldots, Y_{\sigma(n)}\right)$ have the same law. (For an introduction to exchangeable sequences and arrays see, e.g., [Aldous 85].) This hypothesis is important for the use of the ensemble to produce statistical null models, as it implies symmetry of the probability distributions with respect to the permutation of variables, i.e., all the nodes or the agents they represent (genes, computer routers, etc.) are given an equivalent status.

To complete the model, one has to specify the choice for $\pi_{n}$, which determines the behavior of the graph ensemble. For example, in previous work [Bassetti et al. 07], we chose the two-parameter distribution

$$
\pi_{n}(d \theta)=Z_{n}^{-1} \theta^{-\beta} \mathbb{I}_{\left(\frac{\alpha}{n}, 1\right]}(\theta) d \theta,
$$


where $n>\alpha>0$ and $\beta>1$ are free parameters, $\mathbb{I}_{\left(\frac{\alpha}{n}, 1\right]}$ is the indicator function of the interval $\left(\frac{\alpha}{n}, 1\right]$, taking the value 1 inside the interval and 0 everywhere else, and $Z_{n}:=\left((n / \alpha)^{\beta-1}-1\right) /(\beta-1)$ is the normalization constant. As we will see in Section 7 , this choice produces a graph ensemble with heavy-tailed degree sequences. As a second example, taking $\pi_{n}(d \theta)=\delta_{\lambda / n}(d \theta)$, one obtains a directed version of the Erdős-Rényi graph.

A naturally interesting problem is to characterize the general forms of the probability measure $\pi_{n}$ that lead to graph ensembles with qualitatively different characteristics. In Section 3 we shall give some results in this direction. Note that a general way of producing the distribution $\pi_{n}$ for each $n$, starting form a given "seed" $F$ ( $F$ being a fixed distribution function on $\mathbb{R}^{+}$), is easily described by the following assumption:

$$
F_{n}(x):=\frac{F(x n)}{F(n)}=\int_{[0, x]} \pi_{n}(d \theta) .
$$

With the above assumption, $F_{n}$ is a well-defined distribution function on $[0,1]$ whenever $F(n)>0$, which certainly holds for large enough values of $n$.

\subsection{Completely Exchangeable Graphs}

The method described in Section 2.1 of generating exchangeable graphs is quite general, so one can imagine many simple variants. For example, one can consider the following algorithm: (i) throw a bias $\theta$ from a prescribed probability distribution $\pi_{n}$, and (ii) set all the elements of $\mathbb{X}_{n}$ to be 0 or 1 according to the toss of a coin with bias $\theta$. The resulting probability law, say $Q$, is

$$
Q\left\{X_{i, j}^{(n)}=e_{i, j} ; i, j=1, \ldots, n\right\}=\int_{[0,1]} \theta^{\sum_{i, j} e_{i, j}}(1-\theta)^{n^{2}-\sum_{i, j} e_{i, j}} \pi_{n}(d \theta) .
$$

For any $e_{i, j}$ in $\{0,1\}, i, j=1, \ldots n$, that is under $Q,\left\{X_{i, j}^{(n)} ; i, j=1, \ldots, n\right\}$ are exchangeable, with de Finetti measure $\pi_{n}$.

\subsection{Hierarchical Models}

Another possible variant considers a hierarchy of probability distributions to generate the bias of the coins. In this case one can take

$$
\begin{aligned}
& Q^{*}\left\{X_{i, j}^{(n)}=e_{i, j}, i=1, \ldots, m_{n}, j=1, \ldots, n\right\}= \\
& \int_{\mathbb{R}^{+}} \prod_{i=1}^{m_{n}} \int_{[0,1]} \theta_{i}^{\sum_{j=1}^{n} e_{i, j}}\left(1-\theta_{i}\right)^{n-\sum_{j=1}^{n} e_{i, j}} \pi_{n}\left(d \theta_{i} \mid \alpha\right) \lambda_{n}(d \alpha),
\end{aligned}
$$


where $\lambda_{n}$ is a probability on $\mathbb{R}^{+}$and $\pi_{n}(d \theta \mid \alpha)$ is a kernel on $[0,1] \times \mathbb{R}^{+}$; that is, for every $\alpha$ in $\mathbb{R}^{+}, \pi_{n}(\cdot \mid \alpha)$ is a measure on the Borel $\sigma$-field of $[0,1]$ and, for every measurable subset $B$ of $[0,1], \alpha \mapsto \pi_{n}(B \mid \alpha)$ is measurable.

\section{Connectivities}

We will continue the main discussion considering the case of partially exchangeable graphs of Section 2.2. Some brief comments on the other variants are reported in Section 6. In the rest of the paper, with the exception of Section 6 , we suppose that all the random elements are defined on the same probability space $(\Omega, \mathcal{F}, P)$ and we denote by $\mathbb{E}(Y)$ the mathematical expectation of a given random variable $Y$ with respect to $P$. With a slight abuse of notation, we shall use indifferently the random graph $G_{n}$ and its adjacency matrix $\mathbb{X}_{n}=\left[X_{i, j}^{(n)}\right]_{i, j}$.

\section{I. In and Out Connectivity}

The first quantities that we want to characterize are the graph degree distributions. The random variable $Z_{m_{n}, j}:=\sum_{i=1}^{m_{n}} X_{i, j}^{(n)}$ represents the in-degree of the $j$ th node in the random graph, while $S_{n, i}:=\sum_{j=1}^{n} X_{i, j}^{(n)}$ can be seen as the out-degree of the $i$ th node $\left(1 \leq i \leq m_{n}\right)$. Note that $\left(Z_{m_{n}, 1}, \ldots, Z_{m_{n}, n}\right)$ are identically distributed as well as $\left(S_{n, 1}, \ldots, S_{n, m_{n}}\right)$. Moreover, $\left(S_{n, 1}, \ldots, S_{n, m_{n}}\right)$ are independent, and each $S_{n, i}$ is a sum of exchangeable Boolean random variables, while $\left(Z_{m_{n}, 1}, \ldots, Z_{m_{n}, n}\right)$ are dependent. Clearly, the mean degrees are equal to $m_{n} \mu_{n}$ and $n \mu_{n}$, respectively, where $\mu_{n}:=P\left\{X_{i, j}^{(n)}=1\right\}=\int_{[0,1]} \theta \pi_{n}(d \theta)$ is the probability of the link $i \rightarrow j$. Note that, while in the Erdős-Rényi model $n \mu_{n}=\lambda$ for every $n$, in this case $n \mu_{n}$ generally depends on $n$. On the other hand, when (2.3) is in force, using the well-known fact that $\mathbb{E}(Y)=\int_{0}^{+\infty}(1-G(y)) d y$ for any positive random variable $Y$ with distribution function $G$, one gets

$$
n \mu_{n}=\int_{[0,1]} n \theta \pi_{n}(d \theta)=\int_{0}^{n}\left(\frac{1-F(x)}{F(n)}\right) d x
$$

and hence, if $\mu:=\int_{0}^{+\infty} x d F(x)<+\infty$, it follows that $n \mu_{n}=\mu+o(1)$. The (marginal) degree distributions are given by

$$
P\left\{S_{n, i}=k\right\}=\left(\begin{array}{c}
n \\
k
\end{array}\right) \int_{[0,1]} \theta^{k}(1-\theta)^{n-k} \pi_{n}(d \theta)
$$

and

$$
P\left\{Z_{m_{n}, j}=k\right\}=\left(\begin{array}{c}
m_{n} \\
k
\end{array}\right) \mu_{n}^{k}\left(1-\mu_{n}\right)^{m_{n}-k} .
$$


With the above expressions, the problem of determining the asymptotic distribution of $\left(Z_{m_{n}, 1}\right)_{n \geq 1}$ and $\left(S_{n, 1}\right)_{n \geq 1}$ is simply cast as a central limit problem for triangular arrays. In fact, while for $\left(Z_{m_{n}, 1}\right)_{n \geq 1}$ a classical central limit theorem (CLT) for triangular arrays of independent random variables works, for $\left(S_{n, i}\right)_{n \geq 1}$ one needs a CLT for exchangeable random variables. General CLTs for exchangeable random variables are well known (see, for instance, [Fortini et al. 96, Regazzini and Sazonov 97]). Here the situation is particularly simple, since we are dealing with zero-one random variables. Consequently, we need only a simple $a d-h o c$ CLT, for exchangeable Boolean random variables.

Let $\tilde{\theta}_{n}$ be a random variable taking values in $[0,1]$ with distribution $\pi_{n}$ and set $T_{n}:=n \tilde{\theta}_{n}$. The next proposition shows that, under a set of reasonable assumptions on $T_{n}$, the limit law of $\left(S_{n, 1}\right)_{n \geq 1}$ is a mixture of Poisson distributions, while the limit law of $\left(Z_{n, 1}\right)_{n \geq 1}$ is a simple Poisson distribution.

Proposition 3.I. (CLT.) If $\left(T_{n}\right)_{n \geq 1}$ converges in distribution to a random variable $T$ with distribution function $F$, then, for every integer $j \geq 1$,

$$
\lim _{n \rightarrow+\infty} P\left\{S_{n, j}=k\right\}=\mathbb{E}\left[\frac{1}{k !} T^{k} e^{-T}\right]=\int_{0}^{+\infty} \frac{t^{k}}{k !} e^{-t} d F(t) \quad(k=0,1, \ldots) .
$$

Moreover, if for some $\lambda>0$ and for a sequence $\left(a_{n}\right)_{n \geq 1}$

$$
\lim _{n \rightarrow+\infty} a_{n} \mathbb{E}\left(T_{n}\right)=\lim _{n \rightarrow+\infty} n a_{n} \int_{[0,1]} \theta \pi_{n}(d \theta)=\lambda
$$

holds true, then, for every integers $k \geq 0$ and $j$,

$$
\lim _{n \rightarrow+\infty} P\left\{Z_{m_{n}, j}=k\right\}=\frac{\lambda^{k} e^{-\lambda}}{k !},
$$

with $m_{n}=\left[n a_{n}\right]([x]$ being the integer part of $x)$.

Remark 3.2. (a) If (2.3) holds true, then the distribution of $T$ is $F$. Indeed, in this case,

$$
\lim _{n \rightarrow+\infty} P\left\{T_{n} \leq x\right\}=\lim _{n \rightarrow+\infty} P\left\{\tilde{\theta}_{n} \leq x / n\right\}=\lim _{n \rightarrow+\infty} \frac{F(x)}{F(n)}=F(x) \quad(x \geq 0) .
$$

(b) It is worth noticing that as a corollary of Theorem 5 in [Fortini et al. 96] one has that the convergence of $T_{n}$ is a necessary and sufficient condition in order to obtain a Poisson mixture as a limit law for $\left(S_{n, j}\right)_{n \geq 1}$. Hence, the first part of the previous proposition can be proved invoking such a theorem. Nevertheless, for the sake of completeness, we shall give here a simple direct proof. 
Proof of Proposition 3.I. Since $T_{n}:=n \tilde{\theta}_{n}$, by (3.1) one has

$$
P\left\{S_{n, j}=k\right\}=\mathbb{E}\left[\left(\begin{array}{l}
n \\
k
\end{array}\right) \frac{1}{n^{k}} T_{n}^{k}\left(1-\frac{T_{n}}{n}\right)^{n(1-k / n)}\right]=\mathbb{E}\left[\phi_{n}\left(T_{n}\right)\right],
$$

where

$$
\phi_{n}(x)=\left(\begin{array}{l}
n \\
k
\end{array}\right) \frac{1}{n^{k}} x^{k}\left(1-\frac{x}{n}\right)^{n(1-k / n)} .
$$

Now,

$$
\mathbb{E}\left[\phi_{n}\left(T_{n}\right)\right]=\mathbb{E}\left[\phi\left(T_{n}\right)\right]+R_{n},
$$

where $\phi(x)=\frac{1}{k !} x^{k} e^{-x}$ and $R_{n}=\mathbb{E}\left[\phi_{n}\left(T_{n}\right)\right]-\mathbb{E}\left[\phi\left(T_{n}\right)\right]$. It is plain to check that $\phi_{n}$ converges uniformly on every compact set to $\phi$. Moreover, since $\left(T_{n}\right)_{n \geq 1}$ converges in distribution, by Prohorov's theorem (see, e.g., [Kallenberg 02, Theorem 16.3]) it should be tight, that is, for every $\epsilon>0$ there exists $K>0$ such that $\sup _{n \geq 1} P\left\{\left|T_{n}\right| \geq K\right\} \leq \epsilon$. Hence, one gets that

$$
\lim _{n \rightarrow+\infty}\left|R_{n}\right| \leq \lim _{n \rightarrow+\infty}\left[\sup _{|x| \leq K}\left|\phi_{n}(x)-\phi(x)\right|+2 P\left\{\left|T_{n}\right| \geq K\right\}\right] \leq 2 \epsilon .
$$

At this stage, the first part of the thesis follows immediately; indeed, $\left(T_{n}\right)_{n \geq 1}$ converges in distribution if and only if $\mathbb{E}\left[f\left(T_{n}\right)\right] \rightarrow \mathbb{E}[f(T)]$ for every bounded continuous function $f$, and $\phi$ is bounded and continuous.

The second part of the thesis follows by the classical Poisson approximation to binomial distribution using (3.2). Indeed,

$$
\mu_{n}=\frac{\lambda}{n a_{n}}(1+o(1))
$$

and $\left[n a_{n}\right]=m_{n}$ with $n a_{n} \rightarrow+\infty$. To see this last fact, observe that, since $T_{n}$ converges in distribution to $T, \tilde{\theta}_{n}$ goes to zero in probability. Using this last fact, it is easy to see that $\mathbb{E} \tilde{\theta}_{n}=\int_{[0,1]} \theta \pi_{n}(d \theta)$ goes to zero, hence $n a_{n}$ must diverge.

Since (3.3) is a mixture of Poisson distributions with weight given by $F$, the above result can be used to "discharge" the choice of $\pi_{n}$ on the perhaps more intuitive choice of the mixing distribution $F$. Clearly, the emergence of heavytailed distributions is not a simple consequence of (2.1) but depends on the choice of $\pi_{n}$. The following example describes a mixing probability that gives rise to a compact out-degree distribution.

Example 3.3. Take

$$
\pi_{n}(d \theta)=\frac{n \gamma}{1-e^{-\gamma n}} e^{-\gamma n \theta} d \theta \quad(\gamma>0)
$$


or, in other words, assume (2.3) with $F(x)=\int_{0}^{x} \gamma e^{-\gamma t} d t=1-e^{-\gamma x}$. With this choice, according to Proposition 3.1, the limit distribution of $S_{n, 1}$ is an exponential mixture of Poisson distribution. Precisely, we find it to be a geometric distribution; indeed,

$$
\begin{aligned}
\lim _{n \rightarrow+\infty} P\left\{S_{n, j}=k\right\} & =\gamma \int_{0}^{+\infty} \frac{t^{k}}{k !} e^{-t} e^{-\gamma t} d t \\
& =\frac{\gamma}{k !(1+\gamma)^{k+1}} \int_{0}^{+\infty} y^{(k+1)-1} e^{-y} d y=\frac{\gamma}{k !(1+\gamma)^{k+1}} \Gamma(k+1) \\
& =\frac{\gamma}{1+\gamma}(1+\gamma)^{-k} \quad(k=0,1, \ldots) .
\end{aligned}
$$

Moreover, $a_{n}=1$ and $\lambda=1 / \gamma$ satisfy the conditions of Proposition 3.1, yielding

$$
\lim _{n \rightarrow+\infty} P\left\{Z_{n, 1}=k\right\}=\frac{\gamma^{-k} e^{-1 / \gamma}}{k !} .
$$

As a generalization of the previous example, take, instead of an exponential distribution, a gamma distribution, i.e.,

$$
F(x)=\int_{0}^{x} \frac{\gamma^{r} t^{r-1} e^{-\gamma t}}{\Gamma(r)} d t \quad(r>0) .
$$

It is easy to check that the limiting distribution is a negative binomial distribution with parameter $r$. That is,

$$
\lim _{n \rightarrow+\infty} P\left\{S_{n, j}=k\right\}=\left(\begin{array}{c}
r+k-1 \\
k
\end{array}\right)\left(\frac{\gamma}{1+\gamma}\right)^{r}(1+\gamma)^{-k} \quad(k=0,1, \ldots) .
$$

Moreover,

$$
\lim _{n \rightarrow+\infty} P\left\{Z_{n, 1}=k\right\}=\frac{\left(\frac{r}{\gamma}\right)^{k} e^{-r / \gamma}}{k !}
$$

In the above example, mixturing the Poisson distribution with exponential weights proves insufficient to produce a power-law distribution. In other instances, a suitable choice of $F$ in (3.3) can give rise to an out-degree probability distribution with heavy tails. Consider the following example.

Example 3.4. Assume a slight generalization of (2.2), i.e.,

$$
\pi_{n}(d \theta)=Z_{n}^{-1} \theta^{-\beta} g(n \theta) \mathbb{I}_{\left(\frac{\alpha}{n}, 1\right]}(\theta) d \theta,
$$

with $0<c_{1} \leq g(\tau) \leq c_{2}<+\infty$ for every $\tau$ in $[0,+\infty)$ and

$$
Z_{n}:=\int_{a / n}^{1} \theta^{-\beta} g(n \theta) d \theta
$$


Note that (3.4) satisfies (2.3) with

$$
F(x)=\frac{\int_{\alpha}^{x} t^{-\beta} g(t) d t}{\int_{\alpha}^{+\infty} t^{-\beta} g(t) d t}
$$

Hence, it is straightforward to verify that Proposition 3.1 yields

$$
\lim _{n \rightarrow+\infty} P\left\{S_{n, j}=k\right\}=\frac{1}{k !} \frac{\int_{\alpha}^{+\infty} t^{k-\beta} e^{-t} g(t) d t}{\int_{\alpha}^{+\infty} t^{-\beta} g(t) d t}=: q_{\alpha, \beta, g}(k) .
$$

We now show that such a distribution is a power-law-tailed distribution. In order to prove this, let us consider first the special case in which $g=1$, i.e., the older (2.2). With this choice, we get

$$
\begin{aligned}
\lim _{n \rightarrow+\infty} P\left\{S_{n, j}=k\right\} & =\frac{\alpha^{\beta-1}(\beta-1)}{k !} \int_{\alpha}^{+\infty} t^{k-\beta} e^{-t} d t \\
& =q_{\alpha, \beta, 1}(k)=: p_{\alpha, \beta}(k) \quad(k \geq 0) .
\end{aligned}
$$

Hence, if $k>\beta$, write

$$
p_{\alpha, \beta}(k)=\alpha^{\beta-1}(\beta-1)\left(\frac{\Gamma(k+1-\beta)}{\Gamma(k+1)}-\frac{1}{\Gamma(k+1)} \int_{0}^{\alpha} t^{k-\beta} e^{-t} d t\right),
$$

and note that, by the well-known asymptotic expansion for the gamma function,

$$
\frac{\Gamma(k+1-\beta)}{\Gamma(k+1)}=\frac{1}{k^{\beta}}(1+o(1)) \quad \text { as } k \rightarrow+\infty .
$$

Moreover,

$$
\frac{k^{\beta}}{\Gamma(k+1)} \int_{0}^{\alpha} t^{k-\beta} e^{-t} d t=o(1) \quad \text { as } k \rightarrow+\infty .
$$

Consequently, we get

$$
p_{\alpha, \beta}(k)=\alpha^{\beta-1}(\beta-1) \frac{1}{k^{\beta}}(1+o(1)) .
$$

Now note that, since

$$
\frac{c_{1}}{c_{2}} p_{\alpha, \beta}(k) \leq q_{\alpha, \beta, g}(k) \leq \frac{c_{2}}{c_{1}} p_{\alpha, \beta}(k),
$$

$k \mapsto q_{\alpha, \beta, g}(k)$ has power-law tails also for $g \neq 1$.

Finally, the following example shows a more complex, already mixtured distribution, leading to a heavy tail. 
Example 3.5. Given $\alpha>1$ and $s>1$, set, for every positive $x$,

$$
f_{\alpha, s}(x):=\frac{1}{\Gamma(s) \Phi(1, s, \alpha)} \int_{0}^{+\infty} e^{-x\left(e^{\tau}-1\right)} \tau^{s-1} e^{-\tau(\alpha-1)} d \tau,
$$

where $\Phi(z, s, \alpha)$ is the well-known Lerch transcendent, defined as $\Phi(z, s, \alpha):=$ $\sum_{k \geq 0} z^{k}(\alpha+k)^{-s}$, for every complex $z$ with $|z| \leq 1$. (See, for instance, [Gradshteyn and Ryzhik 00, 9.550].) Note that $f_{\alpha, s}(x) \geq 0$. Moreover, by means of the following integral representation $\Gamma(s) \Phi(z, s, \alpha)=\int_{0}^{+\infty} \tau^{s-1} e^{-\tau(\alpha-1)}\left(e^{\tau}-z\right)^{-1} d \tau$ (see [Gradshteyn and Ryzhik 00, 9.556]), one can check that $\int_{0}^{+\infty} f_{\alpha, s}(x) d x=1$. In other words, $f_{\alpha, s}$ defines a density distribution. Note that $f_{\alpha, s}$ is itself a mixture of exponential densities. Indeed, it can be rewritten as

$$
\begin{aligned}
f_{\alpha, s}(x) & =\int_{0}^{+\infty}\left(e^{\tau}-1\right) e^{-x\left(e^{\tau}-1\right)} \frac{\tau^{s-1} e^{-\tau(\alpha-1)}}{\Gamma(s) \Phi(1, s, \alpha)\left(e^{\tau}-1\right)} d \tau \\
& =\int_{0}^{+\infty} u e^{-x u} \frac{\log ^{s-1}(u+1)}{\Gamma(s) \Phi(1, s, \alpha)(u+1)^{\alpha+1}} d u
\end{aligned}
$$

with

$$
\int_{0}^{+\infty} \frac{\tau^{s-1} e^{-\tau(\alpha-1)}}{\Gamma(s) \Phi(1, s, \alpha)\left(e^{\tau}-1\right)} d \tau=\int_{0}^{+\infty} \frac{\log ^{s-1}(u+1)}{\Gamma(s) \Phi(1, s, \alpha)(u+1)^{\alpha+1}} d u=1 .
$$

It can be verified, with the help of Fubini's theorem and the already mentioned integral representation of the Lerch transcendent, that for every real $q$ with $|q|<1$

$$
\begin{aligned}
\sum_{k \geq 0}(i q)^{k} \int_{0}^{+\infty} \frac{t^{k}}{k !} e^{-t} f_{\alpha, s}(t) d t & =\int_{0}^{+\infty} e^{i q t} e^{-t} f_{\alpha, s}(t) d t \\
& =\frac{\Phi(i q, s, \alpha)}{\Phi(1, s, \alpha)}=\sum_{k \geq 0} \frac{(i q)^{k}}{\Phi(1, s, \alpha)(\alpha+k)^{s}}
\end{aligned}
$$

(where $i:=\sqrt{-1}$ ), from which it follows that

$$
\int_{0}^{+\infty} \frac{t^{k}}{k !} e^{-t} f_{\alpha, s}(t) d t=\frac{1}{\Phi(1, s, a)(\alpha+k)^{s}} .
$$

Hence, if one takes an exchangeable random graph $G_{n}$, with mixing distribution satisfying (2.3) with

$$
F(x):=\int_{0}^{x} f_{\alpha, s}(t) d t,
$$


then the limit law of $S_{n, 1}$ is given by

$$
\lim _{n \rightarrow+\infty} P\left\{S_{n, 1}=k\right\}=\int_{0}^{+\infty} \frac{t^{k}}{k !} e^{-t} f_{\alpha, s}(t) d t=\Phi(1, s, a)^{-1}(\alpha+k)^{-s}
$$

for every $k \geq 0$.

As Examples 3.3-3.5 show, the model can produce graphs with disparate features, depending on the choice of the probability distribution of the coin biases. In particular, it is interesting to investigate under which conditions do heavytailed distributions emerge as limit distributions of the out-degree. If one supposes that $T_{n}$ converges in law to a random variable with probability distribution function $F$, we have shown how the question can be reduced to the problem of determining under which conditions on $F$ the probability defined by (3.3) has heavy tails. It is worth noticing that mixtures of Poisson distributions have been extensively studied (see, e.g., [Grandell 97]). Let us briefly recall some useful properties of such distributions. First of all, if

$$
p_{k}:=\int_{0}^{+\infty} \frac{1}{k !} t^{k} e^{-t} d F_{i}(t) \quad(k \geq 0, i=1,2)
$$

for two distribution functions $F_{1}$ and $F_{2}$ with $F_{i}(x)=0$ for every $x \leq 0$, then $F_{1}=F_{2}$. (This simple fact was first noticed in [Feller 48]; see also [Grandell 97, Theorem 2.1(i)].) Hence, one hopes to recover many properties of

$$
p_{k}:=\int_{0}^{+\infty} \frac{1}{k !} t^{k} e^{-t} d F(t)
$$

from the properties of $F$. In particular, Theorem 2.1 in [Willmot 90] states that if $F$ has a density $f$ with respect to the Lebesgue measure or to the counting measure, such that

$$
f(x)=L(x) x^{\alpha} \exp \{-\beta x\}(1+o(1)) \quad \text { as } x \rightarrow+\infty,
$$

where $L$ is locally bounded and varies slowly at infinity, $\beta \geq 0,-\infty<\alpha<+\infty$ (with $\alpha<-1$ if $\beta=0$ ), then

$$
p_{k}=L(k) \beta^{-(\alpha+1)}\left(\frac{1}{1+\beta}\right)^{k} k^{\alpha}(1+o(1)) \quad \text { as } k \rightarrow+\infty .
$$

Recall that a slowly varying function $L$ is a measurable function such that

$$
\lim _{x \rightarrow+\infty} L(x t) / L(x)=1
$$


for every positive $t$. Under no assumptions on $F$ we have the following very simple lemma.

Lemma 3.6. Let $F$ be a distribution function with $F(x)=0$ for every $x \leq 0$, and set $p_{k}:=\int_{0}^{+\infty} \frac{1}{k !} t^{k} e^{-t} d F(t)$. Then, for every positive $\gamma$,

$$
\sum_{k \geq 0} k^{\gamma} p_{k}<+\infty
$$

if and only if

$$
\int_{0}^{+\infty} t^{\gamma} d F(t)<+\infty
$$

The proof is deferred to Section 8 .

It is also worth mentioning that a random variable $T$ is a mixture of Poisson distribution if and only if its generating function $G_{T}(s)=\mathbb{E}\left(s^{T}\right)$ is absolutely monotone in $(-\infty, 1)$, that is, if $G_{T}^{(n)}(s) \geq 0$ for every integer $n$ and $s$ in $(-\infty, 1)$. (See [Puri and Goldie 79] and [Grandell 97, Proposition 2.2].) Finally, we recall that the sequence $\left(p_{k}\right)_{k \geq 1}$ inherits many properties from $F$. For example, $\left(p_{k}\right)_{k \geq 1}$ has a monotone density if $F$ has a monotone distribution, $\left(p_{k}\right)_{k \geq 1}$ has log-convex density if $F$ has log-convex distribution, and $\left(p_{k}\right)_{k \geq 1}$ is infinitely divisible if $F$ is so. (For more details see, for instance, [Grandell 97, Steutel and van Harn 04].)

The next subsections will deal with the computation of interesting observables that go beyond the degree distributions.

\subsection{The Hub Size}

As a first example of observable, we discuss the size of the so-called hub, i.e., the node having maximal out-degree among the nodes (thus, in many concrete networks, being the most important for routing and the most vulnerable to attack; see, e.g., [Bollobás and Riordan 03b]). The hub size is defined by the expression

$$
H_{n}:=\max _{i=1, \ldots, m_{n}}\left(S_{n, i}\right) .
$$

In particular, the most interesting case for the behavior of the hub is when the tail of the out-degree is power-law, as this means that there can be no characteristic size for the hub. As we will explain, it is interesting to give an analytical expression of the limit law of this quantity under a suitable rescaling. The idea is very simple: by stochastic independence, it is clear that $P\left\{H_{n} \leq\right.$ $\left.x b_{n}\right\}=\left(1-P\left\{S_{n, 1}>x b_{n}\right\}\right)^{m_{n}}$, where $x>0$ is any positive number. Now, 
after setting $L:=\sup \left\{y \geq 0: \limsup _{n}\left[y b_{n}\right] / n<1\right\}$, if we can prove that $P\left\{S_{n, 1} \leq x b_{n}\right\}=1-g(x) / m_{n}+o\left(1 / m_{n}\right)$ for any $x \leq L$, then

$$
\lim _{n} P\left\{H_{n} \leq x b_{n}\right\}=e^{-g(x)} \mathbb{I}_{[0, L)}(x)+\mathbb{I}_{[L,+\infty)}(x) .
$$

We will show that, in some situations, it is possible to determine explicitly $g$, $b_{n}$, and $L$. The following proposition concerns the hub behavior in case of heavy tails for the out-degree.

Proposition 3.7. Suppose there exist two positive constants $\eta, c_{\eta}$, a sequence of positive numbers $\left(c_{\eta, n}\right)_{n \geq 1}$, and a sequence of functions $\left(r_{n}\right)_{n \geq 1}$, such that for every $t$ in $(0,1)$

$$
\int_{(t, 1]} \pi_{n}(d \theta)=c_{\eta, n} \frac{1}{(n t)^{\eta}}+r_{n}(t)
$$

$c_{\eta, n} \rightarrow c_{\eta}$, and

$$
\frac{\int_{0}^{1} r_{n}(t) t^{\left[b_{n} x\right]}(1-t)^{n-\left[b_{n} x\right]-1} d t}{B\left(\left[b_{n} x\right]+1, n-\left[b_{n} x\right]\right)}=o\left(\frac{1}{m_{n}}\right),
$$

with $b_{n}:=m_{n}^{1 / \eta}$ and $B(\alpha, \beta):=\int_{0}^{1} u^{\alpha-1}(1-u)^{\beta-1} d u$. Then,

$$
\lim _{n \rightarrow+\infty} P\left\{H_{n} \leq\left[x b_{n}\right]\right\}=e^{-c_{\eta} x^{-\eta}} \mathbb{I}_{[0, L)}(x)+\mathbb{I}_{[L,+\infty)}(x),
$$

where

$$
L:=\sup \left\{y \geq 0: \limsup _{n \rightarrow+\infty}\left[y m_{n}^{1 / \eta}\right] / n<1\right\}
$$

Proof. First of all let us start by recalling the well-known relation

$$
\sum_{k=\left[x b_{n}\right]+1}^{n}\left(\begin{array}{l}
n \\
k
\end{array}\right) \theta^{k}(1-\theta)^{n-k}=\frac{\int_{0}^{\theta} t^{\left[b_{n} x\right]}(1-t)^{n-\left[b_{n} x\right]-1} d t}{B\left(\left[b_{n} x\right]+1, n-\left[b_{n} x\right]\right)},
$$

where $B(\alpha, \beta)=\int_{0}^{1} t^{\alpha-1}(1-t)^{\beta-1} d t=\Gamma(\alpha) \Gamma(b) / \Gamma(a+b)$. (See, e.g., [Magnus et al. 66, Section 9.2.5].) Hence, by (3.1), (3.6), and Fubini's theorem, one gets

$$
\begin{aligned}
P\left\{S_{n, 1}>\left[x b_{n}\right]\right\} & =\int_{[0,1]} \sum_{k=\left[x b_{n}\right]+1}^{n}\left(\begin{array}{l}
n \\
k
\end{array}\right) \theta^{k}(1-\theta)^{n-k} \pi_{n}(d \theta) \\
& =\int_{[0,1]} \int_{0}^{\theta} \frac{t^{\left[b_{n} x\right]}(1-t)^{n-\left[b_{n} x\right]-1} d t}{B\left(\left[b_{n} x\right]+1, n-\left[b_{n} x\right]\right)} \pi_{n}(d \theta) \\
& =\frac{1}{B\left(\left[b_{n} x\right]+1, n-\left[b_{n} x\right]\right)} \int_{0}^{1} t^{\left[b_{n} x\right]}(1-t)^{n-\left[b_{n} x\right]-1} F_{n}^{*}(t) d t,
\end{aligned}
$$


with

$$
F_{n}^{*}(t):=\int_{(t, 1]} \pi_{n}(d \theta)=P\left\{\tilde{\theta}_{n}>t\right\} .
$$

Now, by hypothesis,

$$
F_{n}^{*}(t)=c_{\eta, n} \frac{1}{(n t)^{\eta}}+r_{n}(t)
$$

Then,

$$
\begin{aligned}
P\left\{S_{n, 1}>\right. & {\left.\left[x b_{n}\right]\right\}=} \\
& \frac{1}{B\left(\left[b_{n} x\right]+1, n-\left[b_{n} x\right]\right)} \frac{c_{n, \eta}}{n^{\eta}} \int_{0}^{1} t^{\left[b_{n} x\right]-\eta}(1-t)^{n-\left[b_{n} x\right]-1} d t+R_{n}(x)
\end{aligned}
$$

with

$$
R_{n}(x):=\frac{1}{B\left(\left[b_{n} x\right]+1, n-\left[b_{n} x\right]\right)} \int_{[0,1]} r_{n}(t) t^{\left[b_{n} x\right]}(1-t)^{n-\left[b_{n} x\right]-1}=o\left(\frac{1}{m_{n}}\right) .
$$

Finally, using once more the asymptotic expression $\Gamma(n+a) / \Gamma(n+b)=n^{a-b}(1+$ $o(1))$ as $n \rightarrow+\infty$, one obtains

$$
\begin{aligned}
P\left\{S_{n, 1}>\left[x b_{n}\right]\right\} & =\frac{1}{m_{n}}\left(\frac{c_{n, \eta} m_{n}}{n^{\eta}} \frac{\Gamma(n+1) \Gamma\left(\left[b_{n} x\right]+1-\eta\right)}{\Gamma(n+1-\eta) \Gamma\left(\left[b_{n} x\right]+1\right)}+o(1)\right) \\
& =\frac{1}{m_{n}}\left(\left(\frac{b_{n}}{\left[x b_{n}\right]}\right)^{\eta} c_{\eta}+o(1)\right),
\end{aligned}
$$

which is

$$
P\left\{S_{n, 1}>\left[x b_{n}\right]\right\}=\frac{1}{m_{n}}\left[\frac{c_{\eta}}{x^{\eta}}+o(1)\right] .
$$

We give now two simple conditions that imply the validity of (3.5), and can be useful in concrete applications. The first conditions will be used in the example that we spell out in detail in the second part of this paper (Proposition 7.2).

Lemma 3.8. If for some $\alpha>0, C<+\infty$, and $\eta>0$,

$$
\left|r_{n}(t)\right| \leq C\left(\mathbb{I}\{n t<\alpha\}\left(1+(n t)^{-\eta}\right)+n^{-\eta}\right)
$$

then (3.5) holds true provided that $m_{n}$ is such that $m_{n} / n^{\eta}=o(1)$.

Proof. Set $\beta_{n}:=\left[x b_{n}\right]$ and

$$
I_{n}(d)=m_{n} \frac{1}{B\left(\beta_{n}+1+d, n-\beta_{n}\right)} \int_{0}^{\alpha / n} t^{\beta_{n}+d}(1-t)^{n-\beta_{n}-1} d t .
$$


Hence,

$$
\begin{aligned}
m_{n}\left|R_{n}(x)\right| \leq C m_{n}\left\{\frac{1}{B\left(\beta_{n}+1, n-\beta_{n}\right)}\right. \\
\left.\times \int_{0}^{\alpha / n}\left(1+\frac{1}{(n t)^{\eta}}\right) t^{\beta_{n}}(1-t)^{n-\beta_{n}-1} d t+\frac{1}{n^{\eta}}\right\} \\
=C\left\{I_{n}(0)+I_{n}(-\eta) \frac{B\left(\beta_{n}+1-\eta, n-\beta_{n}\right)}{n^{\eta} B\left(\beta_{n}+1, n-\beta_{n}\right)}+o(1)\right\} \\
\leq C\left\{I_{n}(0)+I_{n}(-\eta) \beta_{n}^{-\eta}+o(1)\right\} .
\end{aligned}
$$

It remains to show that $I_{n}(0)+I_{n}(-\eta) \beta_{n}^{-\eta}=o(1)$. With the help of the Sirling formula, one has

$$
\begin{aligned}
I_{n}(d)= & \frac{m_{n} \Gamma(n+1+d)}{\Gamma\left(\beta_{n}+1+d\right) \Gamma\left(n-\beta_{n}\right)} \int_{0}^{\alpha / n} t^{\beta_{n}+d}(1-t)^{n-\beta_{n}-1} d t \\
\leq & m_{n} \frac{m_{n} \Gamma(n+1+d)}{\Gamma\left(\beta_{n}+1+d\right) \Gamma\left(n-\beta_{n}\right)}\left(\frac{\alpha}{n}\right)^{\beta_{n}+d-1} \\
\leq & C_{1} m_{n} \frac{\exp \left\{\log (\alpha)\left(1+d+\beta_{n}\right)\right\}(n+1+d)^{n+d+1 / 2}}{n^{\beta_{n}+d+1}\left(\beta_{n}+1+d\right)^{\beta_{n}+d+1 / 2}\left(n-\beta_{n}\right)^{n-\beta_{n}-1 / 2}} \\
& \quad \times \exp \left\{-(n+1+d)+\beta_{n}+1+d+n-\beta_{n}\right\} \\
= & C_{1} m_{n} \frac{\exp \left\{\log (\alpha)\left(1+d+\beta_{n}\right)\right\}\left(1+\frac{1+d}{n}\right)^{n\left(1+\frac{d+1 / 2}{n}\right)}}{\beta_{n}^{\beta_{n}+d+1 / 2}\left(1+\frac{1+d}{\beta_{n}}\right)^{\beta_{n}\left(1+\frac{d+1 / 2}{b_{n}}\right)}\left(1-\frac{b_{n}}{n}\right)^{n\left(1-\frac{\beta_{n}+1 / 2}{n}\right)}} \\
\leq & \left.C_{2} m_{n} \frac{\exp \left\{\log (\alpha)\left(1+d+\beta_{n}\right)-\log \left(\beta_{n}\right)\left(\frac{1}{2}+d+\beta_{n}\right)\right\}}{n}\right)^{\left.n / \beta_{n}\right]^{\beta_{n}-\beta_{n}^{2} / n}} \\
\leq & C_{3} m_{n} \exp \left\{\log (\alpha)\left(1+d+\beta_{n}\right)-\log \left(\beta_{n}\right)\left(\frac{1}{2}+d+\beta_{n}\right)+\left(\beta_{n}-\frac{\beta_{n}^{2}}{n}\right)\right\} \\
\leq & C_{4} \exp \left\{\log \left(m_{n}\right)-C_{5} \beta_{n} \log \left(\beta_{n}\right)\right\} .
\end{aligned}
$$

Since $\beta_{n}=x^{1 / \eta} m_{n}^{1 / \eta}(1+o(1))$ and $m_{n}^{1 / \eta} / n=o(1)$ the thesis follows easily.

We conclude this subsection observing that when (2.3) is in force,

$$
\int_{(t, 1]} \pi_{n}(d \theta)=1-F_{n}(t)=\frac{F(n)-1+1-F(n t)}{F(n)},
$$

hence it is natural to assume some hypotheses on $1-F(x)$. In particular, recall that a distribution function $F$ is in the domain of attraction of the extreme value Fréchet distribution if and only if

$$
\sup \{x: F(x)<1\}=+\infty \quad \text { and } \quad 1-F(x)=\frac{1}{x^{\eta}} L(x),
$$


where $L$ is a slowly varying function (see [Galambos 87]). This means that (3.7) holds if and only if given a sequence of independent and identically distributed random variables $(\xi)_{n \geq 1}$ with common law $F$,

$$
\lim _{n \rightarrow+\infty} P\left\{a_{n}^{-1} \max \left\{\xi_{1}, \ldots, \xi_{n}\right\} \leq x\right\}=e^{-c_{\eta} x^{-\eta}} \quad(x>0)
$$

for a suitable normalizing sequence $\left(a_{n}\right)_{n}$. In point of fact, (3.7) is not sufficient, in our case, to ensure that $r_{n}$ is a reminder of the right order. Hence, we need a heavier requirement.

Lemma 3.9. Assume that (2.3) is in force with

$$
1-F(x)=\frac{c_{\eta}}{x^{\eta}}[1+h(x)]
$$

for some $\eta>0$ and $0<c_{\eta}<+\infty$, with

$$
|h(x)| \leq A\left(\frac{1}{x^{\delta_{1}}}+\frac{1}{x^{\delta_{2}}}\right) \quad\left(x>0, A<+\infty, \delta_{1}, \delta_{2}>0\right) .
$$

Then, (3.5) holds true with $m_{n} / n^{\eta}=o(1)$.

Proof. Assume that $\delta_{1}=\delta$ and $\delta_{2}=\delta$. In the same notation as in the proof of Proposition 3.7,

$$
r_{n}(t)=\frac{c_{\eta}}{F(n) n^{\eta}}(1+h(n))+\frac{c_{\eta} h(n t)}{F(n)(n t)^{\eta}} .
$$

Now,

$$
\begin{aligned}
R_{n}:=R_{n}(x) & =\frac{1}{B\left(\left[b_{n} x\right]+1, n-\left[b_{n} x\right]\right)} \int_{[0,1]} r_{n}(t) t^{\left[b_{n} x\right]}(1-t)^{n-\left[b_{n} x\right]-1} d t \\
& =R_{n}^{(1)}+R_{n}^{(2)}
\end{aligned}
$$

with

$$
\begin{aligned}
R_{n}^{(1)} & =\frac{c_{\eta}(1+h(n))}{F(n) n^{\eta}} \frac{1}{B\left(\left[b_{n} x\right]+1, n-\left[b_{n} x\right]\right)} \int_{[0,1]} t^{\left[b_{n} x\right]}(1-t)^{n-\left[b_{n} x\right]-1} d t \\
& =\frac{c_{\eta}(1+h(n))}{F(n) n^{\eta}}=o\left(m_{n}^{-1}\right)
\end{aligned}
$$

and

$$
R_{n}^{(2)}=\frac{1}{B\left(\left[b_{n} x\right]+1, n-\left[b_{n} x\right]\right)} \int_{[0,1]} t^{\left[b_{n} x\right]}(1-t)^{n-\left[b_{n} x\right]-1} \frac{c_{\eta} h(n t)}{F(n)(n t)^{\eta}} d t
$$


Finally,

$$
\begin{aligned}
\left|R_{n}^{(2)}\right| & \leq \frac{c_{\eta} 2 A}{n^{\delta+\eta} B\left(\left[b_{n} x\right]+1, n-\left[b_{n} x\right]\right)} \int_{[0,1]} t^{\left[b_{n} x\right]-(\eta+\delta)}(1-t)^{n-\left[b_{n} x\right]-1} d t \\
& =c_{\eta} 2 A \frac{B\left(\left[b_{n} x\right]+1-\eta-\delta, n-\left[b_{n} x\right]\right)}{n^{\delta+\eta} B\left(\left[b_{n} x\right]+1, n-\left[b_{n} x\right]\right)} \\
& =c_{\eta} 2 A \frac{\Gamma\left(\left[b_{n} x\right]+1-\eta-\delta\right) \Gamma(n+1)}{n^{\delta+\eta} \Gamma(n+1-\eta-\delta) \Gamma\left(\left[b_{n} x\right]+1\right)} \\
& \leq c_{\eta} 2 A^{\prime} \frac{(n+1)^{\delta+\eta}}{n^{\delta+\eta}\left(\left[b_{n} x\right]+1\right)^{\delta+\eta}} \leq c_{\eta} 2 A^{\prime} 2^{\delta+\eta}\left(\frac{b_{n}}{\left[b_{n} x\right]}\right)^{\delta+\eta} \frac{1}{b_{n}^{\delta+\eta}} .
\end{aligned}
$$

for a suitable constant $A^{\prime}$; hence, since $b_{n}^{\eta}=m_{n},\left|R_{n}^{(2)}\right|=o\left(m_{n}^{-1}\right)$. The general case follows in the same way.

Example 3.10. As an example it is easy to see that

$$
1-F(x)=\frac{\alpha^{\eta}}{(\alpha+x)^{\eta}} \quad(x \geq 0, \alpha>0, \eta>0)
$$

satisfies the assumption of Lemma 3.9. In point of fact,

$$
1-F(x)=\frac{\alpha^{\eta}}{x^{\eta}}+\frac{\alpha^{\eta}}{x^{\eta}}\left[\frac{x^{\eta}-(\alpha+x)^{\eta}}{(x+\alpha)^{\eta}}\right],
$$

hence

$$
|h(x)| \leq \frac{(\alpha+x)^{\eta}-x^{\eta}}{x^{\eta}} .
$$

If $x \leq 1$, then $|h(x)| \leq(1+\alpha)^{\eta} / x^{\eta}$, while if $x>1$,

$$
|h(x)| \leq\left(1+\frac{\alpha}{x}\right)^{\eta}-1
$$

Since $t \rightarrow t^{\eta}$ is a Lipschitz function of constant $\eta(1+\alpha)^{\eta-1}$ on $[1,1+\alpha]$, if $x>1$, it follows that $(1+\alpha / x)^{\eta}-1 \leq \eta(1+\alpha)^{\eta-1}|1+\alpha / x-1|=\eta(1+\alpha)^{\eta-1} a / x$. In summary,

$$
|h(x)| \leq A\left[\frac{1}{x}+\frac{1}{x^{\eta}}\right] .
$$

\section{Some Nonlocal Features of the Graphs}

In this section, we deal with the subgraphs content and the mean number of roots and leaves of the model of Section 2.1. 


\section{I. Subgraphs}

The simple exchangeable structure of the generated random graphs makes it possible to compute easily the mean value of the number of subgraphs "of a given shape" contained in the graph, which can be used for the discovery of "network motifs" [Shen-Orr et al. 02, Milo et al. 02, Milo et al. 04, Matias 06].

Consider a subgraph, with $k$ nodes and $m$ edges, given by

$$
H=\left\{i_{1} \rightarrow i_{(1,1)}, \ldots, i_{1} \rightarrow i_{\left(1, m_{1}\right)}, i_{2} \rightarrow i_{(2,1)}, \ldots, i_{k} \rightarrow i_{(k, 1)}, \ldots, i_{k} \rightarrow i_{\left(k, m_{k}\right)}\right\}
$$

with $\sum_{i=1}^{k} m_{i}=m$. Of course,

$$
P\left\{H \in G_{n}\right\}=\int_{[0,1]} \theta_{1}^{m_{1}} \pi_{n}\left(d \theta_{1}\right) \int_{[0,1]} \theta_{2}^{m_{2}} \pi_{n}\left(d \theta_{2}\right) \ldots \int_{[0,1]} \theta_{k}^{m_{k}} \pi_{n}\left(d \theta_{k}\right) .
$$

Denote by $\mathfrak{T}$ the set of all subgraphs isomorphic to $H$ contained in the complete $n$ graph and by $N(H)$, the cardinality of such set. Since the number $\mathcal{N}_{H}\left(G_{n}\right)$ of graphs isomorphic to $H$ contained in $G_{n}$ can be clearly written as

$$
\mathcal{N}_{H}\left(G_{n}\right)=\sum_{g \in \mathfrak{T}} \mathbb{I}\left\{g \in G_{n}\right\}
$$

it follows that

$$
\mathbb{E}\left[\mathcal{N}_{H}\left(G_{n}\right)\right]=N(H) P\left\{H \in G_{n}\right\} ;
$$

indeed, by exchangeability, $P\left\{g \in G_{n}\right\}=P\left\{H \in G_{n}\right\}$ for every $g$ in $\mathfrak{T}$.

For example, let us consider the $k$-cycles. A subgraph $H$ is called a $k$-cycle if it has the form $i_{1} \rightarrow i_{2} \rightarrow \cdots \rightarrow i_{k} \rightarrow i_{1}$. If $\mathcal{N}_{C_{k}}\left(G_{n}\right)$ denotes the number of $k$-cycles contained in $G_{n}$, then

$$
\mathbb{E}\left[\mathcal{N}_{C_{k}}\left(G_{n}\right)\right]=2\left(\begin{array}{l}
n \\
k
\end{array}\right) \mu_{n}^{k}
$$

Things are slightly more complicated for rectangular matrices because in the evaluation of $N(H)$ one needs to take into consideration also the constraints given by the fact that only $m_{n}$ nodes can send outgoing edges. In what follows we will discuss mainly the case of square matrices.

As we shall see, in the study of transcriptional networks, the 3 -cycle $i_{1} \rightarrow$ $i_{2}, i_{2} \rightarrow i_{3}, i_{3} \rightarrow i_{1}$ is called the feedback loop ( $\mathrm{fbl}$ ), while the feedforward loop $(\mathrm{ffl})$ is a triangle of the form $i_{1} \rightarrow i_{2} \rightarrow i_{3}, i_{1} \rightarrow i_{3}$. Following the procedure described above, one gets

$$
\mathbb{E}\left[\mathcal{N}_{\mathrm{fbl}}\left(G_{n}\right)\right]=2\left(\begin{array}{c}
n \\
3
\end{array}\right) \mu_{n}^{3}
$$


As for the evaluation of feedforward loops, we have

$$
\mathbb{E}\left[\mathcal{N}_{\mathrm{ffl}}\left(G_{n}\right)\right]=6\left(\begin{array}{l}
n \\
3
\end{array}\right) \int_{[0,1]} \theta^{2} \pi_{n}(d \theta) \int_{[0,1]} \theta \pi_{n}(d \theta) .
$$

It is worth mentioning that, in principle, it is possible to compute analytically the variance, as well as any other moment, of the number of subgraphs isomorphic to a given subgraph. However, computations become lengthy and cumbersome rather soon. As an example, we considered the variance of the number of feedback loops and feedforward loops.

The key point is evaluating $\mathbb{E} \mathcal{N}_{\text {ffl }}\left(G_{n}\right)^{2}$ and $\mathbb{E} \mathcal{N}_{\text {fbl }}\left(G_{n}\right)^{2}$. Again, for the sake of symplicity, we will deal only with square matrices. It is clear that $\mathbb{E} \mathcal{N}_{\mathrm{fbl}}\left(G_{n}\right)^{2}=\sum_{t \in \mathfrak{T}} \sum_{s \in \mathfrak{T}} P\left\{s, t \in G_{n}\right\}$, where $\mathfrak{T}$ is the set of all feedback loops contained in the complete $n$ graph. Analogously one obtains $\mathbb{E} \mathcal{N}_{\text {fll }}\left(G_{n}\right)^{2}$ taking as $\mathfrak{T}$ the set of all feedforward loops. Simple calculations give

$$
\begin{aligned}
\mathbb{E}\left[\mathcal{N}_{\mathrm{fbl}}\left(G_{n}\right)^{2}\right]= & 4\left(\begin{array}{c}
n \\
3
\end{array}\right)\left(\begin{array}{c}
n-3 \\
3
\end{array}\right) \mu_{n}^{6}+12\left(\begin{array}{c}
n \\
3
\end{array}\right)\left(\begin{array}{c}
n-3 \\
2
\end{array}\right) \mu_{n}^{4} \delta_{2, n} \\
& +6(n-3)\left(\begin{array}{c}
n \\
3
\end{array}\right)\left(\mu_{n}^{3} \delta_{2, n}+\mu_{n}^{2} \delta_{2, n}^{2}\right)+2\left(\begin{array}{c}
n \\
3
\end{array}\right)\left(\mu_{n}^{3}+\delta_{2}^{3}\right),
\end{aligned}
$$

where $\delta_{i, n}:=\int_{0}^{1} \theta^{i} \pi_{n}(d \theta)$. As for $\mathcal{N}_{\mathrm{ffl}}$, the computations are longer, but essentially the same. The problem is that $P\left\{s, t \in G_{n}\right\}$ can take many different expressions depending on $s$ and $t$. With straightforward but tedious calculations, one gets

$\mathbb{E}\left[\mathcal{N}_{\mathrm{ffl}}\left(G_{n}\right)^{2}\right]=\left(\begin{array}{l}n \\ 3\end{array}\right) A_{n}+(n-3)\left(\begin{array}{l}n \\ 3\end{array}\right) B_{n}+\left(\begin{array}{c}n \\ 3\end{array}\right)\left(\begin{array}{c}n-3 \\ 2\end{array}\right) C_{n}+\left(\begin{array}{c}n \\ 3\end{array}\right)\left(\begin{array}{c}n-3 \\ 3\end{array}\right) D_{n}$,

with

$$
\begin{aligned}
A_{n}= & 6 \delta_{1, n} \delta_{2, n}+3 \delta_{1, n}^{2} \delta_{2, n}+6 \delta_{1, n} \delta_{2, n}^{2}+3 \delta_{2, n}^{2}+\delta_{2, n}^{3} \\
B_{n}= & 30 \delta_{1, n} \delta_{2, n}^{2}+18 \delta_{1, n}^{2} \delta_{2, n}^{2}+6 \delta_{2, n}^{3}+18 \delta_{1, n}^{2} \delta_{3, n} \\
& +12 \delta_{1, n} \delta_{2, n} \delta_{3, n}+6 \delta_{2, n} \delta_{3, n}+3 \delta_{3, n}^{2} \\
C_{n}= & 60 \delta_{1, n}^{2} \delta_{2, n}^{2}+12 \delta_{2, n}^{3}+24 \delta_{1, n} \delta_{2, n} \delta_{3, n}+12 \delta_{1, n}^{2} \delta_{4, n}, \\
D_{n}= & 36 \delta_{1, n}^{2} \delta_{2, n}^{2} .
\end{aligned}
$$

Hence,

$$
\begin{aligned}
\operatorname{Var}\left(\mathcal{N}_{\mathrm{fbl}}\left(G_{n}\right)\right)= & 12\left(\begin{array}{c}
n \\
3
\end{array}\right)\left(\begin{array}{c}
n-3 \\
2
\end{array}\right) \mu_{n}^{4} \delta_{2, n}+6(n-3)\left(\begin{array}{c}
n \\
3
\end{array}\right)\left(\mu_{n}^{3} \delta_{2, n}+\mu_{n}^{2} \delta_{2, n}^{2}\right) \\
& +2\left(\begin{array}{l}
n \\
3
\end{array}\right)\left(\mu_{n}^{3}+\delta_{2, n}^{3}\right)-4\left(\begin{array}{l}
n \\
3
\end{array}\right) R_{n} \mu_{n}^{6}
\end{aligned}
$$


and

$$
\begin{aligned}
\operatorname{Var}\left(\mathcal{N}_{\mathrm{ffl}}\left(G_{n}\right)\right)= & \left(\begin{array}{l}
n \\
3
\end{array}\right) A_{n}+(n-3)\left(\begin{array}{l}
n \\
3
\end{array}\right) B_{n} \\
& +\left(\begin{array}{c}
n \\
3
\end{array}\right)\left(\begin{array}{c}
n-3 \\
2
\end{array}\right) C_{n}-R_{n} D_{n}
\end{aligned}
$$

with $R_{n}=\left[\left(\begin{array}{l}n \\ 3\end{array}\right)-\left(\begin{array}{c}n-3 \\ 3\end{array}\right)\right]$.

\subsection{Roots and Leaves}

We say that $i$ is a root if there is no edge of the kind $j \rightarrow i$ but there is at least one edge of the kind $i \rightarrow j$ with $j \neq i$. Loops do not count. Conversely, we say that $i$ is a leaf if there is no edge of the kind $i \rightarrow j$ but there is at least one edge of the kind $j \rightarrow i$ with $j \neq i$. Again we exclude loops and isolated points. Let $\mathfrak{L}\left(G_{n}\right)$ be the number of leaves in $G_{n}$ and $\mathfrak{R}(G)$ the number of roots in $G_{n}$. Of course, $\mathfrak{L}\left(G_{n}\right)=\sum_{i=1}^{n} \mathfrak{L}_{i}\left(G_{n}\right)$ and $\mathfrak{R}\left(G_{n}\right)=\sum_{i=1}^{m_{n}} \mathfrak{R}_{i}\left(G_{n}\right)$, where $\mathfrak{L}_{i}\left(G_{n}\right)$ is equal to 1 if $i$ is a leaf of $G_{n}$ and 0 otherwise and, similarly, $\mathfrak{R}_{i}(G)=1$ if $i$ is a root of $G_{n}$ and 0 otherwise. It follows that

$$
\mathfrak{R}_{i}\left(G_{n}\right)=\mathbb{I}\left\{\sum_{j=1}^{m_{n}} X_{j, i}=0\right\}\left(1-\mathbb{I}\left\{\sum_{j=1, i \neq j}^{n} X_{i, j}=0\right\}\right),
$$

and analogously,

$$
\mathfrak{L}_{i}\left(G_{n}\right)=\mathbb{I}\left\{\sum_{j=1}^{n} X_{i, j}=0\right\}\left(1-\mathbb{I}\left\{\sum_{j=1, j \neq i}^{m_{n}} X_{j, i}=0\right\}\right) .
$$

Hence,

$$
\mathbb{E}\left[\mathfrak{L}_{i}\left(G_{n}\right)\right]=\left(1-\mu_{n}\right)^{m_{n}}\left(1-P\left\{S_{n-1, i}=0\right\}\right)
$$

and

$$
\mathbb{E}\left[\Re_{i}\left(G_{n}\right)\right]=\left(1-\left(1-\mu_{n}\right)^{m_{n}-1}\right) P\left\{S_{n, i}=0,\right\}
$$

and then

$$
\begin{aligned}
\mathbb{E}\left[\mathfrak{L}\left(G_{n}\right)\right] & =n\left(1-\mu_{n}\right)^{m_{n}}\left(1-P\left\{S_{n-1, i}=0\right\}\right), \\
\mathbb{E}\left[\Re_{i}\left(G_{n}\right)\right] & =m_{n}\left(1-\left(1-\mu_{n}\right)^{m_{n}-1}\right) P\left\{S_{n, i}=0\right\} .
\end{aligned}
$$

\subsection{Connected Components}

One of the classic and most studied problems in the mathematics of random graphs is the existence and the size of the so-called giant component (see, for 
instance, [Bollobás and Riordan 04b, Chung and Lu 06b, Chung and Lu 03, Chung and Lu 06a, Durrett 07] and references therein). This is, in principle, an important property if one wants to use the ensemble as a null or positive model for a real-world system. In many empirical instances, such as the Internet, the World Wide Web, and many biological networks, the existence of a very large component can be observed directly. For this reason, if this property is absent, a model could have limited applications. Of course, in our model the existence of a giant component depends on the choice of the measure $\pi_{n}$. A detailed study of this problem is beyond the scope of this work, and it will be dealt with in future papers. While, for the moment, we did not prove any general theorem, in some interesting case, such as the power-law model defined by (2.2), one can study the problem numerically. In this case, our simulations indicate the emergence of a giant component for all values of the parameters, which makes the model attractive for applications (see also [Bassetti et al. 07]). On general grounds, it is not hard to see that for this example the probability that $G_{n}$ has only one connected component goes to zero as $n$ diverges (at least for $\beta>2$ and square matrices). This is a consequence of a more general proposition.

Proposition 4.I. Let $m_{n}=n$ and assume that $\lim _{n \rightarrow+\infty}\left(1-\mu_{n}\right)^{n-1} P\left\{S_{n, i}=0\right\}=$ $a>0$, then

$$
\lim _{n \rightarrow+\infty} P\left\{G_{n} \text { is connected }\right\}=0 .
$$

Proof. If $Y(n)=\sum_{i=1}^{n} Y_{i, n}$ with $Y_{i, n}=\mathbb{I}\left\{S_{n, i}=0, Z_{n, i}=0\right\}$, then

$$
P\left\{G_{n} \text { is connected }\right\} \leq P\{Y(n)=0\} \leq \frac{\operatorname{Var}(Y(n))}{\mathbb{E}\left(Y^{2}(n)\right)}=1-\frac{\mathbb{E}(Y(n))^{2}}{\mathbb{E}\left(Y^{2}(n)\right)} .
$$

Since $\mathbb{E}(Y(n))=n \mathbb{E}\left(Y_{1, n}\right)=n P\left\{S_{n, i}=0, Z_{n, i}=0\right\}$ and

$$
\begin{aligned}
\mathbb{E}\left(Y(n)^{2}\right)= & n \mathbb{E}\left(Y_{1, n}^{2}\right)+n(n-1) \mathbb{E}\left(Y_{1, n} Y_{2, n}\right) \\
= & n P\left\{S_{n, 1}=0, Z_{n, 1}=0\right\} \\
& +n(n-1) P\left\{S_{n, 1}=0, Z_{n, 1}=0, S_{n, 2}=0, Z_{n, 2}=0\right\} \\
= & n\left(1-\mu_{n}\right)^{n-1} P\left\{S_{n, 1}=0\right\}+n(n-1)\left(1-\mu_{n}\right)^{2 n-2} P\left\{S_{n, 1}=0\right\}^{2},
\end{aligned}
$$

we get

$$
\begin{aligned}
& P\left\{G_{n} \text { is connected }\right\} \\
& \quad \leq 1-\frac{\left(1-\mu_{n}\right)^{2 n-2} P\left\{S_{n, 1}=0\right\}^{2}}{\frac{n-1}{n}\left(1-\mu_{n}\right)^{2 n-2} P\left\{S_{n, 1}=0\right\}^{2}+\frac{1}{n}\left(1-\mu_{n}\right)^{n-1} P\left\{S_{n, 1}=0\right\}} .
\end{aligned}
$$

Taking the limit for $n \rightarrow+\infty$ gives the thesis. 


\section{Threshold Properties in the Kernel of $\boldsymbol{A}_{n}$}

Another interesting facet of the exchangeable graph ensemble is its connection with the theory of systems of random equations over finite algebraic structures.

This problem has fairly important applications in the theory of finite-state automata, the theory of coding, cryptography, and combinatorial optimization problems (satisfiability, coloring). Problems of this kind arise in many branches of science, ranging from statistical physics (theory of glasses) to information theory (e.g., low-density parity-check codes). (See, e.g., [Erdős and Rényi 64, Erdős and Rényi 68, Kolchin 99, MacKay 99, Mézard et al. 02, Mézard et al. 03, Murayama and Okada 03, Levitskaya 05].)

One interesting problem in random linear systems over finite algebraic structures is to prove a threshold property for the random graph $G_{n}$ with adjacency matrix $\mathbb{X}_{n}$ of dimension $m_{n} \times n$. More precisely, one aims to prove that if $m_{n}$ and $n$ diverge with $n / m_{n} \rightarrow \gamma \leq 1$, then an abrupt change in the behavior of the rank of the matrix $\mathbb{X}_{n}$ occurs when the parameter $\gamma$ exceeds a "critical" value $\gamma_{c}$. This property can be expressed in terms of the total number of hypercycles in $G_{n}$, defined as

$$
\mathcal{S}\left(\mathbb{X}_{n}\right)=2^{\operatorname{Ker}\left(\mathbb{X}_{n}\right)}-1=2^{n-m_{n}} \mathcal{N}\left(\mathbb{X}_{n}\right)-1,
$$

where $\mathcal{N}\left(\mathbb{X}_{n}\right)$ is the number of nontrivial (i.e., nonzero) solutions of the linear system in $\mathbb{G F}_{2}$ (the field with elements 0 and 1 )

$$
\mathbb{X}_{n}^{T} x=\mathbb{G F}_{\mathbb{F}_{2}} 0
$$

Problems of this kind have been extensively studied for a few ensembles of random graphs. (See, for instance, [Kolchin 99, Theorem 3.5.1] and [Levitskaya 05, Theorem 1].)

In the next proposition, we give an exact expression for the mean value of the number of solutions of the linear system (5.2). This expression can be used to prove the existence of a threshold property for $\mathcal{S}\left(\mathbb{X}_{n}\right)$. Moreover, the same expression is a first step for a more exhaustive characterization of solution space, which shall be dealt with in a forthcoming paper. All the proofs of this section are deferred to Section 8 .

In order to state the next proposition, we introduce the following notations. Define

$$
\xi_{n}(i)=\int_{[0,1]}(1-2 \theta)^{i} \pi_{n}(d \theta)
$$

and

$$
\mathcal{Z}_{n}=\left\{j \in\{0,1, \ldots, n\} \mid \xi_{n}(j)=0\right\}
$$


Proposition 5.I. Assume that $\mathbb{X}_{n}$ is a random adjacency matrix of dimension $m_{n} \times n$ with law (2.1). Then,

$$
\mathbb{E} \mathcal{N}\left(\mathbb{X}_{n}\right)=2^{-n} \sum_{i=1}^{n}\left(\begin{array}{c}
n \\
i
\end{array}\right)\left(1+\xi_{n}(i)\right)^{m_{n}}
$$

whenever $\mathcal{Z}_{n}$ is the empty set.

Using the previous result one easily obtains the following large deviation estimate.

Proposition 5.2. If $m_{n}=\left[\frac{n}{\gamma}\right](\gamma \leq 1)$ and $\left(T_{n}\right)_{n \geq 1}$ converges in distribution to a random variable $T$ with distribution function $F$, then

$$
\lim _{n \rightarrow+\infty} \frac{1}{n} \log \left(\mathbb{E} \mathcal{N}\left(\mathbb{X}_{n}\right)\right)=\sup _{x \in[0,1]} \Theta_{\gamma}(x)=: I_{\gamma},
$$

with

$$
\begin{aligned}
\Theta_{\gamma}(x):= \\
\frac{1}{\gamma}\left[\log \left(1+\int_{[0,+\infty)} e^{-2 x t} d F(t)\right)-\gamma(x \log (x)+(1-x) \log (1-x)+\log (2))\right],
\end{aligned}
$$

whenever $\mathcal{Z}_{n}$ is the empty set for $n$ large enough.

Combining the previous result with (5.1), it is clear that, under the hypotheses of Proposition 5.2, the mean number of hypercycles $\mathbb{E} \mathcal{S}\left(\mathbb{X}_{n}\right)$ can be written as

$$
\begin{aligned}
\mathbb{E} \mathcal{S}\left(\mathbb{X}_{n}\right) & =(1+o(1))\left(2^{n-m_{n}} e^{n I_{\gamma}} P_{n}-1\right) \\
& =(1+o(1))\left(\exp \left\{n\left(I_{\gamma}-\left(\frac{1}{\gamma}-1\right) \log (2)\right)\right\} P_{n}-1\right),
\end{aligned}
$$

where $P_{n}$ is a function of $n$ that is at most polynomial, i.e., $1 / n \log \left(P_{n}\right)=o(1)$ (as $n \rightarrow+\infty)$. Hence, if $I_{\gamma}>(1 / \gamma-1) \log (2)$, it follows that $\mathbb{E} \mathcal{S}\left(\mathbb{X}_{n}\right)$ diverges exponentially in $n$ as $n$ goes to $+\infty$, while if $I_{\gamma}=(1 / \gamma-1) \log (2)$, it is subexponential, that is, for some $b \geq 0, \mathbb{E} \mathcal{S}\left(\mathbb{X}_{n}\right) / n^{b}$ goes to zero as $n$ diverges.

In point of fact, we have the following lemma.

Lemma 5.3. If $\int_{[0,+\infty)} t d F(t)<+\infty$, then

$$
\sup _{x \in[0,1]} \Theta_{\gamma}(x)>\Theta_{\gamma}(0)=\left(\frac{1}{\gamma}-1\right) \log (2) .
$$


If

$$
\log (x)\left(\int_{[0,+\infty)} t e^{-2 x t} d F(t)\right)^{-1}=o(1) \quad(x \rightarrow 0),
$$

then there exists a $\gamma_{c}$ such that for any $\gamma \leq \gamma_{c}$

$$
\sup _{x \in[0,1]} \Theta_{\gamma}(x)=\Theta_{\gamma}(0)=\left(\frac{1}{\gamma}-1\right) \log (2),
$$

while for $\gamma>\gamma_{c}$

$$
\sup _{x \in[0,1]} \Theta_{\gamma}(x)>\Theta_{\gamma}(0)=\left(\frac{1}{\gamma}-1\right) \log (2) .
$$

In particular, if

$$
1-F(t)=t^{-\beta} L(t)
$$

with $0<\beta<1$ and $L$ a slowly varying function, then (5.3) holds true.

In other words, under the hypotheses of Proposition 5.2, if (5.3) holds true, then there exists a constant $0<\gamma_{c}<1$ such that

$$
\lim _{n \rightarrow+\infty} \frac{\mathbb{E} \mathcal{S}\left(\mathbb{X}_{n}\right)}{n^{b}}= \begin{cases}0 & \text { for some } b=b(\gamma) \geq 0 \text { if } \gamma \leq \gamma_{c}, \\ +\infty & \text { for every } b \geq 0 \text { if } \gamma>\gamma_{c} .\end{cases}
$$

That is, the above mentioned threshold property holds.

\section{Other Models}

In this short section we give some comments about the other two models presented in Sections 2.2-2.3.

\section{I. Completely Exchangeable Graphs}

Most of the properties and quantities discussed in Sections 3, 4, and 5 can be easily established for the totally exchangeable case. Again, $\mu_{n}:=Q\left\{X_{i, j}^{(n)}=1\right\}=$ $\int_{[0,1]} \theta \pi_{n}(d \theta)$ and, for instance, the degree distributions (for a square adjacency matrix) are given by

$$
Q\left\{S_{n, i}=k\right\}=Q\left\{Z_{n, j}=k\right\}=\left(\begin{array}{l}
n \\
k
\end{array}\right) \int_{[0,1]} \theta^{k}(1-\theta)^{n-k} \pi_{n}(d \theta) .
$$

Hence, for instance, we have the following proposition. 
Proposition 6.I. If $\left(T_{n}\right)_{n \geq 1}$ converges in distribution to a random variable $T$ with distribution function $F$, then, for every integer $j \geq 1$,

$$
\lim _{n \rightarrow+\infty} Q\left\{S_{n, j}=k\right\}=\mathbb{E}\left[\frac{1}{k !} T^{k} e^{-T}\right]=\int_{0}^{+\infty} \frac{t^{k}}{k !} e^{-t} d F(t) \quad(k=0,1, \ldots)
$$

and

$$
\lim _{n \rightarrow+\infty} Q\left\{Z_{n, j}=k\right\}=\mathbb{E}\left[\frac{1}{k !} T^{k} e^{-T}\right]=\int_{0}^{+\infty} \frac{t^{k}}{k !} e^{-t} d F(t) \quad(k=0,1, \ldots) .
$$

For this model, quantities such as the mean number of subgraphs, roots, and leaves are again easily computed analytically along the same lines described in Section 4. For example, for motifs

$$
Q\left\{H \in G_{n}\right\}=\int_{[0,1]} \theta^{m} \pi(d \theta)
$$

when

$$
H=\left\{i_{1} \rightarrow i_{(1,1)}, \ldots, i_{1} \rightarrow i_{\left(1, m_{1}\right)}, i_{2} \rightarrow i_{(2,1)}, \ldots, i_{k} \rightarrow i_{(k, 1)}, \ldots, i_{k} \rightarrow i_{\left(k, m_{k}\right)}\right\}
$$

with $\sum_{i=1}^{k} m_{i}=m$. Hence,

$$
\mathbb{E}_{Q}\left(\mathcal{N}_{H}\left(G_{n}\right)\right)=N(H) Q\left\{H \in G_{n}\right\}
$$

Finally, throwing triangular matrices with the same algorithm, one can easily generate models for undirected graphs.

\subsection{Hierarchical Models}

One interesting use of this variant is that it can be exploited to produce directed graphs having power-law tailed in- and out-degree distributions with different exponents. To illustrate this point, we will consider the following example.

Example 6.2. If $\gamma>\beta>2, A>0, \lambda_{n}(d \alpha) \propto \mathbb{I}_{[A, n / 2]} \alpha^{-\gamma} d \alpha$ and $\pi_{n}(d \theta \mid \alpha) \propto$ $\mathbb{I}_{(\alpha / n, 1]} \theta^{-\beta} d \theta$, then $Q^{*}\left\{S_{n, 1}=k\right\}=c_{1} k^{-\beta}(1+o(1))$ and $Q^{*}\left\{Z_{n, 1}=k\right\}=$ $c_{2} k^{-\gamma}(1+o(1))$. Indeed, it is easy to check (by means of a usual dominated convergence argument) that

$$
\lim _{k \rightarrow+\infty} Q^{*}\left\{S_{n, 1}=k\right\}=\frac{(\beta-1)(\gamma-1) A^{\gamma-1}}{k !} \int_{A}^{+\infty} \int_{\alpha}^{+\infty} \alpha^{\beta-\gamma-1} t^{k-\beta} e^{-t} d t d \alpha
$$


and, moreover,

$$
\begin{aligned}
\frac{(\beta-1)(\gamma-1) A^{\gamma-1}}{k !} \int_{A}^{+\infty} \int_{\alpha}^{+\infty} \alpha^{\beta-\gamma-1} t^{k-\beta} & e^{-t} d t d \alpha \\
& =\frac{\gamma-1}{\gamma-\beta} p_{A, \beta}(k)-\frac{\beta-1}{\gamma-\beta} p_{A, \gamma}(k) .
\end{aligned}
$$

In the same way it is easy to check that $\lim _{k \rightarrow+\infty} Q^{*}\left\{Z_{n, 1}=k\right\}=p_{u, \gamma}(k)$ with $u=A(\beta-1) /(\beta-2)$.

\section{A Simple Two-Parameter Model}

In this section, we focus our attention on random graphs generated by assuming (2.2), and we shall specialize the results of previous sections to this twoparameter model. This model has been suggested by a biological application. Hence, before presenting the results, we briefly recall the main features of a transcription network.

Transcription networks are directed graphs that represent regulatory interactions between genes. Specifically, the link $a \rightarrow b$ exists if the protein coded by gene $a$ affects the transcription of gene $b$ in mRNA formed by binding along DNA in a site upstream of its coding region [Babu et al. 04]. For a few organisms, such as E. coli and S. cerevisiae, a significant fraction of the wiring diagram of this network is known [Lee et al. 02, Guelzim et al. 02, Salgado et al. 01, Harbison et al. 04]. The topological features of the graphs can be studied to infer information on the large-scale architecture and evolution of gene regulation in living systems. For instance, the connectivity and the clustering coefficient have been considered [Guelzim et al. 02]. For this kind of analysis, one has to consider null ensembles of random networks with some topological invariant compared to the empirical case. The idea behind it is to establish when and to what extent the empirical topology deviates from the "typical case" statistics of the null ensemble. For example, a topological feature that has led to relevant biological findings, in particular for transcription, is the occurrence of small subgraphs - or "network motifs" [Milo et al. 02, Milo et al. 04, Wolf and Arkin 03, Yeger-Lotem et al. 04].

As usual in statistical studies, the choice of the invariant properties for the randomized counterpart is delicate. For instance, the null ensemble used for motif discovery usually conserves the degree sequences of the original network. The observed degree sequences for the known transcription networks roughly follow a power-law distribution for the out-degree, with exponent between one and two, while being Poissonian in the in-degree [Guelzim et al. 02, Cosentino 
Lagomarsino et al. 05]. These features suggest considering also alternative null models for directed random graphs with Poisson in-degree distribution and (approximately) power-law out-degree distribution, which can be easily generated with our model under (2.2). In the remainder of the paper, we will discuss this case in more detail, showing explicit calculations of the observables discussed in the previous sections.

\section{I. In and Out Connectivity}

By simple calculations from (2.2), we get the following:

- If $1<\beta<2$, then

$$
\mu_{n}=\frac{(\beta-1) \alpha^{\beta-1}}{(2-\beta) n^{\beta-1}} \frac{1-\left(\frac{\alpha}{n}\right)^{2-\beta}}{1-\left(\frac{\alpha}{n}\right)^{1-\beta}}=\frac{1}{n^{\beta-1}} \frac{\alpha^{\beta-1}(\beta-1)}{2-\beta}[1+o(1)] \quad \text { as } n \rightarrow+\infty \text {. }
$$

- If $\beta=2$, then

$$
\mu_{n}=\frac{\alpha}{n-\alpha}(\log n-\log \alpha)=\frac{\log n}{n} \alpha[1+o(1)] \quad \text { as } n \rightarrow+\infty .
$$

- If $\beta>2$, then

$$
\mu_{n}=\frac{(\beta-1)}{(\beta-2)} \frac{\left(\frac{n}{\alpha}\right)^{\beta-2}-1}{\left(\frac{n}{\alpha}\right)^{\beta-1}-1}=\frac{1}{n} \frac{\alpha(\beta-1)}{\beta-2}[1+o(1)] \quad \text { as } n \rightarrow+\infty .
$$

The next proposition, which is a consequence of Proposition 3.1, shows that $S_{n, i}$ is asymptotically power-law distributed, while $Z_{n, i}$, at least with a suitable choice of $m_{n}$, is asymptotically Poisson distributed. One has to distinguish among the different possible scalings for $\mu_{n}$. More precisely, we have the following.

Proposition 7.I. Assume that (2.2) holds true. Then, for every $\alpha>0$ and $\beta>1$,

$$
\lim _{n \rightarrow+\infty} P\left\{S_{n, j}=k\right\}=p_{\alpha, \beta}(k) \quad(j>0, k \geq 0) .
$$

Moreover, if $\beta>2$ and $m_{n}=[\delta n](\delta \in(0,1],[y]$ being the integer part of $y)$, then

$$
\lim _{n \rightarrow+\infty} P\left\{Z_{m_{n}, j}=k\right\}=\frac{e^{-\lambda} \lambda^{k}}{k !} \quad(j>0, k \geq 0),
$$

where $\lambda=\frac{\delta \alpha(\beta-1)}{(\beta-2)}$. If $\beta=2$ and $m_{n}=[\delta n / \log (n)]$, then

$$
\lim _{n \rightarrow+\infty} P\left\{Z_{m_{n}, j}=k\right\}=\frac{e^{-\delta \alpha}(\delta \alpha)^{k}}{k !} \quad(j>0, k \geq 0) .
$$


If $1<\beta<2$ and $m_{n}=\left[\delta n^{\beta-1}\right]$, then

$$
\lim _{n \rightarrow+\infty} P\left\{Z_{m_{n}, j}=k\right\}=\frac{e^{-\lambda} \lambda^{k}}{k !} \quad(j>0, k \geq 0),
$$

where $\lambda=\frac{\delta \alpha^{\beta-1}(\beta-1)}{(2-\beta)}$.

It is worth noticing that asking for a degree distribution that brings to an out-degree having a power-law tail with divergent mean $(\beta \leq 2)$ poses a heavy constraint on the number of regulator nodes (the rows of the matrix).

\subsection{Subgraphs}

We will discuss mainly the case of square matrices, where calculations are simpler and conceptually equivalent.

7.2.I. $\quad \boldsymbol{k}$-cycles. Under (2.2), using (4.1), if $\beta>2$, then

$$
\lim _{n \rightarrow+\infty} \frac{1}{2} \mathbb{E}\left(\mathcal{N}_{C_{k}}\left(G_{n}\right)\right)=\frac{1}{k !}\left[\frac{\alpha(\beta-1)}{(\beta-2)}\right]^{k} ;
$$

if $\beta=2$, then

$$
\lim _{n \rightarrow+\infty} \frac{1}{2(\log n)^{k}} \mathbb{E}\left(\mathcal{N}_{C_{k}}\left(G_{n}\right)\right)=\frac{\alpha^{k}}{k !}
$$

and if $1<\beta<2$, then

$$
\lim _{n \rightarrow+\infty} \frac{1}{2 n^{k(2-\beta)}} \mathbb{E}\left(\mathcal{N}_{C_{k}}\left(G_{n}\right)\right)=\frac{1}{k !}\left(\frac{(\beta-1) \alpha^{\beta-1}}{2-\beta}\right)^{k} .
$$

7.2.2. Triangles. The feedforward loop is a classical example of "network motif," i.e., it is overrepresented in known transcription networks. Conversely, feedback loops (which in principle could form switches and oscillators) are usually underrepresented ("anti-motifs") in transcription networks [Shen-Orr et al. 02, Milo et al. 04].

Here, we evaluate, for our model, the mean number of feedback loops versus feedforward loops. Under (2.2), (4.2) yields

$$
\mathbb{E} \mathcal{N}_{\mathrm{ffl}}\left(G_{n}\right)=6\left(\begin{array}{l}
n \\
3
\end{array}\right) \frac{(\beta-1)^{2}}{\left[\left(\frac{n}{\alpha}\right)^{\beta-1}-1\right]^{2}} \int_{\alpha / n}^{1} \theta^{2-\beta} d \theta \int_{\alpha / n}^{1} \theta^{1-\beta} d \theta
$$

Hence, if $\beta>3$, then

$$
\lim _{n \rightarrow+\infty} \mathbb{E}\left(\mathcal{N}_{\text {ffl }}\left(G_{n}\right)\right)=\frac{(\beta-1)^{2} \alpha^{3}}{(\beta-3)(\beta-2)}>3 \lim _{n \rightarrow+\infty} \mathbb{E}\left(\mathcal{N}_{\text {fbl }}\left(G_{n}\right)\right)=\frac{(\beta-1)^{3} \alpha^{3}}{(\beta-2)^{3}}
$$


if $\beta=3$, then

$$
\lim _{n \rightarrow+\infty} \frac{1}{\log n} \mathbb{E}\left(\mathcal{N}_{\text {ffl }}\left(G_{n}\right)\right)=\alpha^{3}
$$

if $2<\beta<3$, then

$$
\lim _{n \rightarrow+\infty} \frac{1}{n^{3-\beta}} \mathbb{E}\left(\mathcal{N}_{\mathrm{ffl}}\left(G_{n}\right)\right)=\frac{\alpha^{\beta}(\beta-1)^{2}}{(\beta-1)(3-\beta)}
$$

if $\beta=2$, then

$$
\lim _{n \rightarrow+\infty} \frac{1}{n \log n} \mathbb{E}\left(\mathcal{N}_{\mathrm{ffl}}\left(G_{n}\right)\right)=\alpha^{2}
$$

and finally, if $1<\beta<2$, then

$$
\lim _{n \rightarrow+\infty} \frac{1}{n^{5-2 \beta}} \mathbb{E}\left(\mathcal{N}_{\text {ffl }}\left(G_{n}\right)\right)=\frac{\alpha^{2 \beta-2}(\beta-1)^{2}}{(3-\beta)(2-\beta)} .
$$

At this stage one can give the scaling behavior of the ratio of the mean number of feedback and feedforward loops, which is

$$
\frac{\mathbb{E} \mathcal{N}_{\mathrm{ffl}}\left(G_{n}\right)}{\mathbb{E} \mathcal{N}_{\mathrm{fbl}}\left(G_{n}\right)} \sim \begin{cases}n^{\beta-1} & \text { if } 1<\beta<2, \\ n /(\log n)^{2} & \text { if } \beta=2, \\ n^{3-\beta} & \text { if } 2<\beta<3, \\ \log n & \text { if } \beta=3, \\ \lambda & \text { if } \beta>3,\end{cases}
$$

where $\lambda=3(\beta-2)^{2}(\beta-3)^{-1}(\beta-1)^{-1}>1$. Here and in what follows we use $a_{n} \sim b_{n}$ to denote $a_{n}=b_{n}(1+o(1))$ as $n \rightarrow+\infty$. Thus, the ffl always dominates, although there is a wide range of regimes. Note that the dominance of feedforward triangles is even stronger if one considers the rectangular adjacency matrices discussed in Section 7.1. For example, for $1<\beta<2$ and rectangular matrices with $m_{n}=n^{\beta-1}$, we calculate

$$
\frac{\mathbb{E} \mathcal{N}_{\text {ffl }}\left(G_{n}\right)}{\mathbb{E} \mathcal{N}_{\text {fbl }}\left(G_{n}\right)} \sim n
$$

As for the variances, for instance, one obtains

$$
\operatorname{Var}\left(\mathcal{N}_{\mathrm{fbl}}\left(G_{n}\right)\right) \sim \begin{cases}n^{5(2-\beta)} & \text { if } 1<\beta<2, \\ (\log n)^{4} & \text { if } \beta=2, \\ \frac{1}{3}\left(\alpha \frac{\beta-1}{\beta-2}\right)^{3} & \text { if } \beta \geq 2 .\end{cases}
$$




\subsection{Roots and Leaves}

By simple computations, from (4.4) we obtain the following:

- If $1<\beta<2$, then

$$
\lim _{n} \frac{1}{n^{2-\beta}} \log \left[\mathbb{E}\left(\Re_{i}\left(G_{n}\right)\right)\right]=-\frac{\beta-1}{2-\beta} \alpha^{\beta-1}
$$

and hence $\mathbb{E}\left(\Re_{i}\left(G_{n}\right)\right) \sim e^{-\frac{\beta-1}{2-\beta} \alpha^{\beta-1} n^{2-\beta}}$.

- If $\beta=2$, then

$$
\lim _{n} \frac{1}{\log n} \log \left[\mathbb{E}\left(\Re_{i}\left(G_{n}\right)\right)\right]=-\alpha
$$

and hence $\mathbb{E}\left(\Re_{i}\left(G_{n}\right)\right) \sim \frac{1}{n^{\alpha}}$.

- If $\beta>2$, then

$$
\lim _{n} \mathbb{E}\left(\Re_{i}\left(G_{n}\right)\right)=\left(1-e^{-\frac{\beta-1}{\beta-2} \alpha}\right) p_{\alpha, \beta}(0) .
$$

Analogously, from (4.3) we derive the following:

- If $1<\beta<2$, then

$$
\lim _{n} \frac{1}{n^{2-\beta}} \log \left[1-\mathbb{E}\left(\mathfrak{L}_{i}\left(G_{n}\right)\right) p_{\alpha, \beta}(0)^{-1}\right]=-\frac{\beta-1}{2-\beta} \alpha^{\beta-1}
$$

and hence $\mathbb{E}\left(\mathfrak{L}_{i}\left(G_{n}\right)\right) \sim\left(1-e^{-\frac{\beta-1}{2-\beta} \alpha^{\beta-1} n^{2-\beta}}\right) p_{\alpha, \beta}(0)$.

- If $\beta=2$, then

$$
\lim _{n} \frac{1}{\log n} \log \left[1-\mathbb{E}\left(\mathfrak{L}_{i}\left(G_{n}\right)\right) p_{\alpha, \beta}(0)^{-1}\right]=-\alpha
$$

and hence $\mathbb{E}\left(\mathfrak{L}_{i}\left(G_{n}\right)\right) \sim\left(1-\frac{1}{n^{\alpha}}\right) p_{\alpha, \beta}(0)$.

- If $\beta>2$, then

$$
\lim _{n} \mathbb{E}\left(\mathfrak{L}_{i}\left(G_{n}\right)\right)=\left(1-e^{-\frac{\beta-1}{\beta-2} \alpha}\right)\left(1-p_{\alpha, \beta}(0)\right) .
$$

Combining all the previous statements, we get $\mathbb{E}\left(\mathfrak{L}\left(G_{n}\right)\right) \sim n$ while

$$
\mathbb{E}\left(\mathfrak{R}\left(G_{n}\right)\right) \sim \begin{cases}e^{-\lambda^{2} n^{2-\beta}} & \text { if } 1<\beta<2, \\ n^{1-\alpha} & \text { if } \beta=2, \\ n & \text { if } \beta>2,\end{cases}
$$

where $\lambda^{2}=\frac{\beta-1}{2-\beta} \alpha^{\beta-1}$.

In concrete applications, these properties can be used, for example, to impose a well-defined scaling for the roots-to-leaves ratio of the null network ensemble. 


\subsection{The Hub}

In Section 3.2, we already explored the implications on the limit laws of the maximally connected node of a power-law distributed out-degree. Using that result under $(2.2)$, it is possible to prove an explicit limit theorem for the size of the hub.

Proposition 7.2. For $\beta>2$ and for every positive number $x$,

$$
\lim _{n \rightarrow+\infty} P\left\{\frac{H_{n}}{b_{n}} \leq x\right\}=e^{-(\alpha / x)^{\beta-1}}
$$

with $m_{n}=n$ and $b_{n}=n^{1 /(\beta-1)}$. For $\beta=2$ and for every positive number $x$,

$$
\lim _{n \rightarrow+\infty} P\left\{\frac{H_{n}}{b_{n}} \leq x\right\}=e^{-(\alpha / x)^{\beta-1}}
$$

with $m_{n}=b_{n}=n / \log n$. Finally, for $1<\beta<2$ and $m_{n}=n^{\beta-1}$,

$$
\lim _{n \rightarrow+\infty} P\left\{\frac{H_{n}}{n} \leq x\right\}=e^{-(\alpha / x)^{\beta-1}} \mathbb{I}_{(0,1)}(x)+\mathbb{I}_{[1, \infty)}(x)
$$

for every positive $x$.

Remark 7.3. (a) Recall that $e^{-(\alpha / x)^{\beta-1}} \mathbb{I}_{[0,+\infty)}(x)$ is the Frechet type II extreme value distribution, that is, one of the three kinds of extreme value distributions that can arise from the limit law of the maximum of independent and identically distributed random variables.

(b) Note that in the last case the limit distribution is not exactly of extreme value kind and the probability of finding a hub of size $n$ is asymptotically finite and equal to $1-e^{-(\alpha)^{\beta-1}}$. This concentration effect was already noted [Itzkovitz et al. 03] for another kind of random graphs ensemble.

Proof of Proposition 7.2. Let $\beta>2$. In the same notation of the proof of Proposition 3.7,

$$
F_{n}^{*}(t)=\mathbb{I}\left\{\frac{\alpha}{n} \leq t\right\} \frac{\alpha^{\beta-1}}{n^{\beta-1}-\alpha^{\beta-1}}\left(t^{1-\beta}-1\right)+\mathbb{I}\left\{\frac{\alpha}{n}>t\right\},
$$

hence $\eta=\beta-1$,

$$
c_{n, \gamma}=\frac{\alpha^{\beta-1}}{1-n^{1-\beta}} \rightarrow c_{\gamma}=\alpha^{\beta-1}
$$

and

$r_{n}(t)=\mathbb{I}\left\{\frac{\alpha}{n}>t\right\}\left(1-\frac{1}{(n t)^{\beta-1}} \frac{\alpha^{\beta-1}}{1-(\alpha / n)^{\beta-1}}\right)-\mathbb{I}\left\{\frac{\alpha}{n} \leq t\right\} \frac{1}{n^{\beta-1}} \frac{\alpha^{\beta-1}}{1-(\alpha / n)^{\beta-1}}$. 
The thesis follows from Proposition 3.7 and Lemma 3.8, noticing that

$$
\left|r_{n}(t)\right| \leq C\left(\left(\frac{1}{(n t)^{\beta-1}}+1\right) \mathbb{I}\left\{\frac{\alpha}{n}>t\right\}+\frac{1}{n^{\beta-1}}\right) .
$$

Arguing essentially in the same way, one can prove the statements for $\beta \leq 2$.

For $\beta>2$ one can guess that $\mathbb{E}\left[H_{n}\right] \sim n^{1 /(\beta-1)}$, as claimed in the analysis of another scale-free random graph ensemble [Itzkovitz et al. 03]. In point of fact, we have the following proposition.

Proposition 7.4. If $\beta>2$ and $d$ is such that $\beta-d>1$, then

$$
\lim _{n \rightarrow+\infty} \mathbb{E}\left[n^{-d /(\beta-1)} H_{n}^{d}\right]=(\beta-1)^{2} \alpha^{2} \Gamma\left(\frac{\beta-1-d}{\beta-1}\right) .
$$

Proof. We begin with the case $d=1$. In Proposition 7.2 we proved that $\left(Y_{n}\right)_{n \geq 1}:=$ $\left(H_{n} / n^{\gamma}\right)_{n \geq 1}$ converges in distribution with $\gamma=1 /(\beta-1)$. So, it is enough to prove that $\left(Y_{n}\right)_{n \geq 1}$ is uniformly integrable, i.e.,

$$
\lim _{L \rightarrow+\infty} \sup _{n} \mathbb{E}\left[\left|Y_{n}\right| \mathbb{I}_{\left|Y_{n}\right| \geq L}\right]=0 .
$$

(See, for instance, [Kallenberg 02, Lemma 4.11].) Note, first, that

$$
\mathbb{E}\left[\left|Y_{n}\right| \mathbb{I}_{\left|Y_{n}\right| \geq L}\right] \leq L P\left\{\frac{H_{n}}{n^{\gamma}}>L\right\}+\int_{L}^{+\infty}\left(1-P\left\{\frac{H_{n}}{n^{\gamma}} \leq x\right\}\right) d x .
$$

Now, by (7.1),

$$
L P\left\{\frac{H_{n}}{n^{\gamma}}>L\right\} \leq C_{1} L\left(1-e^{-\alpha^{\beta-1} L^{1-\beta}}\right)
$$

for a suitable constant $C_{1}$. Hence, $\lim _{L \rightarrow+\infty} \sup _{n} L P\left\{H_{n} / n^{\gamma}>L\right\}=0$. As for the second term, setting $F_{S_{n}}(x)=P\left\{S_{n} \leq x\right\}$, one has

$$
\begin{aligned}
1-P\left\{\frac{H_{n}}{n^{\gamma}} \leq x\right\}= & 1-\left[F_{S_{n}}\left(x n^{\gamma}\right)\right]^{n} \\
= & 1-\exp \left\{n \log \left(F_{S_{n}}\left(x n^{\gamma}\right)\right)\right\} \\
& \quad\left[\operatorname{using} 1-e^{x} \leq-x\right] \\
\leq & -n \log \left(1-\left(1-F_{S_{n}}\left(x n^{\gamma}\right)\right)\right) \\
& \leq\left(1+C_{2}\right) n\left(1-F_{S_{n}}\left(x n^{\gamma}\right)\right) .
\end{aligned}
$$

Hence,

$$
\int_{L}^{+\infty}\left(1-P\left\{\frac{H_{n}}{n^{\gamma}} \leq x\right\}\right) d x \leq\left(1+C_{2}\right) \int_{L}^{+\infty} n\left(1-F_{S_{n}}\left(x n^{\gamma}\right)\right) d x=: I_{n}
$$


since $1-F_{S_{n}}\left(x n^{\gamma}\right)=0$ if $x n^{\gamma}>n$, that is, if $x \geq n^{\frac{\beta-2}{\beta-1}}$,

$$
I_{n}=\frac{(\beta-1)\left(1+C_{2}\right) n}{n^{\beta-1}\left(\frac{1}{\alpha^{\beta-1}}-\frac{1}{n^{\beta-1}}\right)} \int_{L}^{n^{\beta-2} \beta-1} \int_{\alpha / n}^{1} \sum_{k=\left[x n^{\gamma}\right]+1}^{n}\left(\begin{array}{l}
n \\
k
\end{array}\right) \theta^{k-\beta}(1-\theta)^{n-k} d \theta d x
$$

Now, if $L>(\beta-1) / n^{\gamma}$, then $\left[x n^{\gamma}\right]+1>0$ for every $x>L$; hence,

$$
\begin{aligned}
I_{n} & \leq C_{3} n^{2-\beta} \int_{L}^{n^{\beta-2} \beta-1} \sum_{k=\left[x n^{\gamma}\right]+1}^{n}\left(\begin{array}{l}
n \\
k
\end{array}\right) B(n-k+1, k-\beta+1) d x \\
& =C_{3} n^{2-\beta} \int_{L}^{n^{\beta-2} \beta-1} \frac{\Gamma(n+1)}{\Gamma(n-\beta+2)} \sum_{k=\left[x n^{\gamma}\right]+1}^{n} \frac{\Gamma(k-\beta+1)}{\Gamma(k+1)} d x \\
& \leq C_{4} n \int_{L}^{n^{\beta-2} \beta-1} \sum_{k=\left[x n^{\gamma}\right]+1}^{n} \frac{1}{k^{\beta}} d x
\end{aligned}
$$

at least for $L$ large enough. Since,

$$
\sum_{k=M}^{n} \frac{1}{k^{\beta}} \leq \int_{M-1}^{n} \frac{1}{x^{\beta}} d x
$$

it follows that

$$
I_{n} \leq C_{4} \int_{L}^{+\infty}\left(\frac{1}{\left[x n^{\gamma}\right]}\right) d x \leq C_{5} \frac{1}{(L-1)^{\beta-2}}
$$

The proof of the case with $d>1$ follows an identical procedure, with $x^{1 / d}$ in place of $x$ and $L^{1 / d}$ in place of $L$.

\subsection{Random Linear System in $\mathbb{G}_{\mathbf{F}}$}

Under (2.2) one has

$$
F(x)=\alpha^{\beta-1}(\beta-1) \int_{\alpha}^{x} \frac{1}{t^{\beta}} d t=\left(1-\frac{\alpha^{\beta-1}}{x^{\beta-1}}\right) \quad(x>\alpha) .
$$

Hence, applying Lemma 5.3, one has that, if $1<\beta<2$, then there exists a constant $\gamma_{c}(\beta)$ such that

$$
\lim _{n \rightarrow+\infty} \frac{\mathbb{E} \mathcal{S}\left(\mathbb{X}_{n}\right)}{n^{b}}= \begin{cases}0 & \text { for some } b=b(\gamma) \text { if } \gamma \leq \gamma_{c}(\beta), \\ +\infty & \text { for every } b \geq 0 \text { if } \gamma>\gamma_{c}(\beta) .\end{cases}
$$

While, if $\beta>2$, no threshold property holds since $\int_{0}^{+\infty} x d F(x)<+\infty$. 


\section{Appendix}

Proof of Lemma 3.6. Let $k>\gamma$, where $k$ is an integer. By hypothesis,

$$
\frac{\Gamma(k+1)}{\Gamma(k-\gamma+1)} p_{k}=\int_{0}^{+\infty} \frac{t^{k-\gamma}}{\Gamma(k-\gamma+1)} e^{-t} d G(t),
$$

where $G(x)=\int_{(0, x]} t^{\gamma} d F(t)$. Summing both sides on $k$, one can write

$$
\sum_{k=[\gamma]+1}^{M+[\gamma]+1} \frac{\Gamma(k+1)}{\Gamma(k-\gamma+1)} p_{k}=\int_{0}^{+\infty} \phi_{\gamma, M}(t) e^{-t} d G(t)
$$

with

$$
\phi_{\gamma, M}(t):=\sum_{k=[\gamma]+1}^{M+[\gamma]+1} \frac{t^{k-\gamma+1}}{\Gamma(k-\gamma+1)}=\sum_{m=0}^{M} \frac{t^{m+\nu_{\gamma}}}{\Gamma\left(m+\nu_{\gamma}+1\right)}
$$

and $\nu_{\gamma}:=[\gamma]+1-\gamma$. Hence,

$$
\sum_{m=0}^{M} \frac{\Gamma(m+[\gamma]+2)}{\Gamma(m+\nu+1)} p_{m+1+[\gamma]}=\int_{0}^{+\infty} \phi_{\gamma, M}(t) e^{-t} d G(t) .
$$

Now, for every $t>0$ and $\nu_{\gamma}$ in $(0,1)$, by 5.2.7.20 in [Prudnikov et al. 86], one has

$$
\lim _{M \rightarrow+\infty} \phi_{\gamma, M}(t)=\sum_{m=0}^{+\infty} \frac{t^{m+\nu_{\gamma}}}{\Gamma\left(m+\nu_{\gamma}+1\right)}=g\left(\nu_{\gamma}, t\right) e^{t},
$$

where

$$
g\left(\nu_{\gamma}, x\right)=\frac{1}{\Gamma\left(\nu_{\gamma}\right)} \int_{0}^{x} \tau^{\nu_{\gamma}-1} e^{-\tau} d \tau
$$

Moreover, $\phi_{\gamma, M}(t) \geq 0$ and the convergence is clearly monotone. Hence, taking the limit as $M$ goes to $+\infty$ in (8.1), by monotone convergence one obtains

$$
\sum_{m=0}^{+\infty} \frac{\Gamma(m+[\gamma]+2)}{\Gamma\left(m+\nu_{\gamma}+1\right)} p_{m+1+[\gamma]}=\int_{0}^{+\infty} g\left(\nu_{\gamma}, t\right) t^{\gamma} d F(t)
$$

with $\int_{0}^{+\infty} g\left(\nu_{\gamma}, t\right) t^{\gamma} d F(t)<+\infty$ if and only if

$$
\sum_{m=0}^{+\infty} \Gamma(m+[\gamma]+2) \Gamma\left(m+\nu_{\gamma}+1\right)^{-1} p_{m+1+[\gamma]}<+\infty .
$$

Now, since $g\left(\nu_{\gamma}, x\right)$ is a distribution function, one has $\int_{0}^{+\infty} g\left(\nu_{\gamma}, t\right) t^{\gamma} d F(t)<+\infty$ if and only if $\int_{0}^{+\infty} t^{\gamma} d F(t)<+\infty$. Moreover, since

$$
\Gamma(m+[\gamma]+2) \Gamma\left(m+\nu_{\gamma}+1\right)^{-1}=(m+[\gamma]+1)^{\gamma} p_{m+1+[\gamma]}(1+o(1))
$$


as $m \rightarrow+\infty$,

$$
\sum_{m=0}^{+\infty} \Gamma(m+[\gamma]+2) \Gamma\left(m+\nu_{\gamma}+1\right)^{-1} p_{m+1+[\gamma]}<+\infty
$$

if and only if

$$
\sum_{m=0}^{+\infty}(m+[\gamma]+1)^{\gamma} p_{m+1+[\gamma]}<+\infty
$$

which proves the lemma.

Proof of Proposition 5.I. Denote by $M\left(m_{n}, n\right)$ the set of all $m_{n} \times n$ adjacency matrices. The number of solutions of linear system $\mathbb{X}_{n}^{T} x=\mathbb{G F}_{2} 0$ is defined as

$$
\mathcal{N}\left(\mathbb{X}_{n}\right)=\sum_{x \in \mathbb{G F}_{2}^{m_{n}}} \mathbb{I}\left\{\mathbb{X}_{n}^{T} x=_{\mathbb{G F}_{2}} 0\right\}
$$

Now note that

$$
\mathbb{I}\left\{x=\mathbb{G F}_{2} 0\right\}=\frac{1+(-1)^{x}}{2},
$$

and write

$$
\mathbb{E} \mathcal{N}\left(\mathbb{X}_{n}\right)=\sum_{A_{n} \in M\left(m_{n}, n\right)} P\left\{\mathbb{X}_{n}=A_{n}\right\} \sum_{x \in \mathbb{G F}_{2}^{m_{n}}} \prod_{j=1}^{n} \frac{1+(-1)^{\sum_{i=1}^{m_{n}}\left(A_{n}\right)_{i j} x_{i}}}{2}
$$

Using (2.1), rewrite the last expression as

$$
\begin{aligned}
& \mathbb{E} \mathcal{N}\left(\mathbb{X}_{n}\right) 2^{-n} \sum_{x \in \mathbb{G F}_{2}^{m_{n}}} \sum_{A_{n} \in M\left(m_{n}, n\right)} \int_{[0,1]^{m_{n}}}\left[\prod_{i=1}^{m_{n}} \pi_{n}\left(d \theta_{i}\right)\right]\left[\prod_{j=1}^{n}\left(1+(-1)^{\sum_{i=1}^{m_{n}}\left(A_{n}\right)_{i j} x_{i}}\right)\right] \\
& \times {\left[\prod_{j=1}^{n} \prod_{i=1}^{m_{n}} \theta_{i}^{\left(A_{n}\right)_{i j}}\left(1-\theta_{i}\right)^{1-\left(A_{n}\right)_{i j}}\right] } \\
&=2^{-n} \sum_{x \in \mathbb{G F}_{2}^{m_{n}}} \int_{[0,1]^{m_{n}}} \prod_{i=1}^{m_{n}} \pi_{n}\left(d \theta_{i}\right)\left[\prod_{j=1}^{n} \sum_{\left(A_{n}\right)_{j} \in \mathbb{G F}_{2}^{m_{n}}}\left(1+(-1)^{\sum_{i=1}^{m_{n}}\left(A_{n}\right)_{i j} x_{i}}\right)\right. \\
&\left.\times \prod_{i=1}^{m_{n}} \theta_{i}^{\left(A_{n}\right)_{i j}}\left(1-\theta_{i}\right)^{1-\left(A_{n}\right)_{i j}}\right]
\end{aligned}
$$

where $\left(A_{n}\right)_{j}=\left\{\left(A_{n}\right)_{1 j}, \ldots,\left(A_{n}\right)_{m_{n} j}\right\}$ and $\left(A_{n}\right)_{i j}$ is the element in position $(i, j)$ of matrix $A_{n}$. Since the above expression in square brackets is independent 
of $j, \mathbb{E} \mathcal{N}\left(\mathbb{X}_{n}\right)$ can be written as

$$
\begin{aligned}
\mathbb{E} \mathcal{N}\left(\mathbb{X}_{n}\right)= & 2^{-n} \sum_{x \in \mathbb{G F}_{2}^{m_{n}}} \int_{[0,1]^{m_{n}}}\left[\prod_{i=1}^{m_{n}} \pi_{n}\left(d \theta_{i}\right)\right] \\
& \times\left[\sum_{a \in \mathbb{G F}_{2}^{m_{n}}}\left(1+(-1)^{\sum_{i=1}^{m_{n}} a_{i} x_{i}}\right) \prod_{i=1}^{m_{n}} \theta_{i}^{a_{i}}\left(1-\theta_{i}\right)^{1-a_{i}}\right]^{n} .
\end{aligned}
$$

At this stage note that

$$
\sum_{a \in \mathbb{G F}_{2}^{m_{n}}} \prod_{i=1}^{m_{n}} \theta_{i}^{a_{i}}\left(1-\theta_{i}\right)^{1-a_{i}}=1
$$

and then

$$
\begin{aligned}
\mathbb{E} \mathcal{N}\left(\mathbb{X}_{n}\right)= & 2^{-n} \sum_{x \in \mathbb{G F}_{2}^{m_{n}}} \int_{[0,1]^{m_{n}}}\left[\prod_{i=1}^{m_{n}} \pi_{n}\left(d \theta_{i}\right)\right] \\
& \times\left[1+\sum_{a \in \mathbb{G F}_{2}^{m_{n}}}(-1)^{\sum_{i=1}^{m_{n}} a_{i} x_{i}} \prod_{i=1}^{m_{n}} \theta_{i}^{a_{i}}\left(1-\theta_{i}\right)^{1-a_{i}}\right]^{n} \\
= & 2^{-n} \sum_{x \in \mathbb{G F}_{2}^{m_{n}}} \int_{[0,1]^{m_{n}}}\left[\prod_{i=1}^{m_{n}} \pi_{n}\left(d \theta_{i}\right)\right] \\
& \times\left[1+\prod_{i=1}^{m_{n}} \sum_{a_{i} \in \mathbb{G F}_{2}}\left((-1)^{x_{i}} \theta_{i}\right)^{a_{i}}\left(1-\theta_{i}\right)^{1-a_{i}}\right]^{n} .
\end{aligned}
$$

After summing over $a_{i}$, we have

$$
\mathbb{E} \mathcal{N}\left(\mathbb{X}_{n}\right)=2^{-n} \sum_{x \in \mathbb{G F}_{2}^{m_{n}}} \int_{[0,1]^{m_{n}}}\left[\prod_{i=1}^{m_{n}} \pi_{n}\left(d \theta_{i}\right)\right]\left[1+\prod_{i=1}^{m_{n}}\left(1-\theta_{i}\left(1-(-1)^{x_{i}}\right)\right)\right]^{n}
$$

Now, using

$$
\mathbb{I}\left\{\bar{x}==_{\mathbb{G F}_{2}} 0\right\}=\frac{1-(-1)^{x}}{2},
$$

where $\bar{x}=x+1$ in $\mathbb{G F}_{2},(8.2)$ can be written as

$$
\mathbb{E} \mathcal{N}\left(\mathbb{X}_{n}\right)=2^{-n} \sum_{x \in \mathbb{G F}_{2}^{m_{n}}} \int_{[0,1]^{m_{n}}}\left[\prod_{i=1}^{m_{n}} \pi_{n}\left(d \theta_{i}\right)\right]\left[1+\prod_{i=1}^{m_{n}}\left(1-2 \theta_{i} \mathbb{I}\left\{\bar{x}_{i}=\mathbb{G F}_{2} 0\right\}\right)\right]^{n}
$$


Moreover, since

$$
\left(1-2 \theta_{i} \mathbb{I}\left\{\bar{x}_{i}==_{\mathbb{G F}_{2}} 0\right\}\right)=\left(1-2 \theta_{i}\right)^{\mathbb{I}\left\{\bar{x}_{i}=\mathbb{G F}_{2}=0\right\}},
$$

we can rewrite the mean number as

$$
\mathbb{E} \mathcal{N}\left(\mathbb{X}_{n}\right)=2^{-n} \sum_{x \in \mathbb{G F}_{2}^{m}} \int_{[0,1]^{m_{n}}}\left[\prod_{i=1}^{m_{n}} \pi_{n}\left(d \theta_{i}\right)\right]\left[1+\prod_{i=1}^{m_{n}}\left(1-2 \theta_{i}\right)^{\mathbb{I}\left\{\bar{x}_{i}=\mathbb{G F}_{2} 0\right\}}\right]^{n}
$$

After the expansion of the last square bracket, we obtain

$$
\begin{aligned}
\mathbb{E} \mathcal{N}\left(\mathbb{X}_{n}\right) & =2^{-n} \sum_{j=1}^{n}\left(\begin{array}{l}
n \\
j
\end{array}\right) \prod_{i=1}^{m_{n}} \sum_{x_{i} \in \mathbb{G F}_{2}} \int_{[0,1]} \pi_{n}\left(d \theta_{i}\right)\left(1-2 \theta_{i}\right)^{\mathbb{I}\left\{\bar{x}_{i}=\mathbb{G F}_{2} 0\right\} j} \\
& =2^{-n} \sum_{j=1}^{n}\left(\begin{array}{l}
n \\
j
\end{array}\right) \prod_{i=1}^{m_{n}} \sum_{x_{i} \in \mathbb{G F}_{2}} \xi_{n}\left(\mathbb{I}\left\{\bar{x}_{i}={ }_{\mathbb{G F}_{2}} 0\right\} j\right) .
\end{aligned}
$$

Finally, it is easy to see that the last sum is independent of $i$. Then,

$$
\begin{aligned}
\mathbb{E} \mathcal{N}\left(\mathbb{X}_{n}\right) & =2^{-n} \sum_{j=1}^{n}\left(\begin{array}{c}
n \\
j
\end{array}\right)\left[\sum_{\sigma \in \mathbb{G F}_{2}} \xi_{n}\left(\mathbb{I}\left\{\bar{\sigma}=\mathbb{G F}_{2} 0\right\} j\right)\right]^{m_{n}} \\
& =2^{-n} \sum_{j=1}^{n}\left(\begin{array}{c}
n \\
j
\end{array}\right)\left(1+\xi_{n}(j)\right)^{m_{n}}
\end{aligned}
$$

Proof of Proposition 5.2. First of all, observe that

$$
\mathbb{E} \mathcal{N}\left(\mathbb{X}_{n}\right)=\sum_{j=1}^{n} 2^{-n}\left(\begin{array}{l}
n \\
j
\end{array}\right) \exp \left\{n \psi_{n}\left(\frac{j}{n}\right)\right\}
$$

where

$$
\psi_{n}(x)=\frac{m_{n}}{n} \log \left(1+\xi_{n}(x n)\right)=\frac{m_{n}}{n} \log \left(1+\mathbb{E}\left[\left(1-\frac{2 T_{n}}{n}\right)^{x n}\right]\right) .
$$

Now recall that one of the most classical examples of a large deviation estimate is

$$
\begin{aligned}
\lim _{M \rightarrow+\infty} \frac{1}{M} \log \left(\sum_{j=0}^{M}\right. & \left.\left(\begin{array}{c}
M \\
j
\end{array}\right) e^{M f_{M}(j / M)}\right) \\
& =\sup _{x \in[0,1]}[f(x)-\{x \log (x)+(1-x) \log (1-x)+\log (2)\}]
\end{aligned}
$$


whenever $\lim _{M \rightarrow+\infty} \sup _{x \in[0,1]}\left|f_{M}(x)-f(x)\right|=0, f$ being a continuous function on $[0,1]$. (See, e.g., [Ellis 06, Theorems 7.1 and 10.2].) Hence, the thesis follows if we prove that for every $K<+\infty$

$$
\lim _{n \rightarrow+\infty} \sup _{|x| \leq K}\left|\psi_{n}(x)-\frac{1}{\gamma} \log \left(1+\int_{[0,+\infty)} e^{-2 x t} d F(t)\right)\right|=0
$$

To prove (8.3) it is enough to prove that for every $K<+\infty$

$$
\lim _{M \rightarrow+\infty} \sup _{|x| \leq K}\left|\mathbb{E}\left[\left(1-\frac{2 T_{M}}{M}\right)^{M x}-e^{-2 T x}\right]\right|=0 .
$$

Since $T_{M}$ converges weakly to $T$ and $e^{-t}$ is a bounded and continuous function on $[0,+\infty)$, then

$$
\lim _{M \rightarrow+\infty} \mathbb{E}\left|e^{-2 T_{M}}-e^{-2 T}\right|=0
$$

Moreover we claim that

$$
\lim _{M \rightarrow+\infty} \mathbb{E}\left|\left(1-\frac{2 T_{M}}{M}\right)^{M}-e^{-2 T_{M}}\right|=0 .
$$

To prove this last claim, set $\phi_{n}(x)=\left(1-\frac{x}{n}\right)^{n}$ and note that $\phi_{n}$ converges uniformly on every compact set to $e^{-x}$. Hence, given $K$,

$$
\lim _{M \rightarrow+\infty} \sup _{|x| \leq K}\left|\phi_{M}(x)-e^{-x}\right|=0 .
$$

Moreover, since $\left(T_{M}\right)_{M \geq 1}$ is tight, for every $\epsilon$ there exists $K>0$ such that $\sup _{M \geq 1} P\left\{\left|T_{M}\right| \geq K\right\} \leq \epsilon$. Now, $\left|\left(1-\frac{2 T_{M}}{M}\right)^{M}-e^{-2 T_{M}}\right| \leq 2$, and then

$$
\begin{aligned}
\lim _{M \rightarrow+\infty} \mathbb{E}\left|\left(1-\frac{2 T_{M}}{M}\right)^{M}-e^{-2 T_{M}}\right| \\
\leq \lim _{M \rightarrow+\infty}\left[\sup _{|x| \leq K}\left|\phi_{M}(x)-\phi(x)\right|+2 P\left\{\left|T_{M}\right| \geq K\right\}\right] \leq 2 \epsilon .
\end{aligned}
$$

That is (8.6). Finally, given $a, b$ in $[-1,1]$ and $x>0$,

$$
\left|a^{x}-b^{x}\right| \leq \sup _{y \in[-1,1]}\left|\frac{d}{d y} y^{x}\right||a-b|=x|a-b| ;
$$


hence, since $0<T_{M} \leq M$, one has $-1 \leq 1-\frac{2 T_{M}}{M} \leq 1$ and then

$$
\begin{aligned}
\mid \mathbb{E}[(1- & \left.\left.\frac{2 T_{M}}{M}\right)^{M x}-e^{-2 T x}\right] \mid \\
& \leq \mathbb{E}\left|\left(1-\frac{2 T_{M}}{M}\right)^{M x}-e^{-2 T_{M} x}\right|+\mathbb{E}\left|e^{-2 T_{M} x}-e^{-2 T x}\right| \\
& \leq|x|\left\{\mathbb{E}\left|\left(1-\frac{2 T_{M}}{M}\right)^{M}-e^{-2 T_{M}}\right|+\mathbb{E}\left|e^{-2 T_{M}}-e^{-2 T}\right|\right\} .
\end{aligned}
$$

Combining (8.5), (8.6), and (8.7), we get (8.4).

Proof of Lemma 5.3. Note that, for every $x$ in $(0,1)$,

$$
\gamma \frac{d}{d x} \Theta_{\gamma}(x)=\frac{2 \int_{[0,+\infty)} t e^{-2 x t} d F(t)}{1+\int_{[0,+\infty)} e^{-2 x t} d F(t)}-\gamma \log x+\gamma \log (1-x)
$$

Hence, if $\int_{[0,+\infty)} t d F(t)<+\infty$, then $\lim _{x \rightarrow 0^{+}} d / d x \Theta(x)=+\infty$ and then $\Theta$ is strictly increasing in a neighborhood of 0 . This last fact implies that

$$
\sup _{x \in[0,1]} \Theta_{\gamma}(x)>\Theta_{\gamma}(0)=\left(\frac{1}{\gamma}-1\right) \log (2) .
$$

If (5.3) holds true, then $\lim _{x \rightarrow 0^{+}} d / d x \Theta_{\gamma}(x)=-\infty$, and hence there exists $\gamma_{c}$ such that for any $\gamma \leq \gamma_{c}$

$$
\sup _{x \in[0,1]} \Theta_{\gamma}(x)=\Theta_{\gamma}(0)=\left(\frac{1}{\gamma}-1\right) \log (2)
$$

Now set

$$
A(x)=\int_{0}^{x} t d F(t) \quad \text { and } \quad H(s):=\int_{0}^{+\infty} t e^{-t s} d F(t)=\int_{0}^{+\infty} e^{-t s} d A(t) .
$$

The well-known Karamata tauberian theorem (see, e.g., [Feller 71]) yields that, given $\sigma>0$ and $L$ slowly varying, $H(s) \sim s^{-\sigma} L(1 / s)$ as $s$ goes to 0 if and only if $A(x) \sim x^{\sigma} L(x) / \Gamma(1+\sigma)$ as $x$ goes to $+\infty$. Hence, it remains to prove that if (5.4) holds true, then $A(x) \sim x^{\sigma} L(x) / \Gamma(1+\sigma)$. Observe that

$$
A(x)=-L(x) x^{1-\beta}+\int_{0}^{x} s^{-\beta} L(s) d s .
$$

At this stage the claim follows since it is easy to check that $\int_{0}^{x} s^{-\beta} L(s) d s=$ $x^{1-\beta} \tilde{L}(x)$, where $\tilde{L}(x)$ is still slowly varying. 
Acknowledgments. We would like to thank Bruno Bassetti for useful discussions and for having encouraged us during this work. We are also grateful to the referees for many helpful comments.

\section{References}

[Aiello et al. 01] W. Aiello, F. Chung, and L. Lu. "A Random Graph Model for Power Law Graphs." Experiment. Math. 10 (2001), 53-66.

[Aiello et al. 02] W. Aiello, F. Chung, and L. Lu. "Random Evolution in Massive Graphs." In Handbook of Massive Data Sets, Massive Computing 4, edited by J. Abello, P. M. Pardalos, and M. G. C. Resende, pp. 97-122. Dordrecht: Kluwer Academic Publishers, 2002.

[Aldous 85] D. Aldous. Exchangeability and Related Topics, Lecture Notes in Mathematics 1117. Berlin: Springer, 1985.

[Babu et al. 04] M. Babu, N. Luscombe, L. Aravind, M. Gerstein, and S. Teichmann. "Structure and Evolution of Transcriptional Regulatory Networks." Curr. Opin. Struct. Biol. 14 (2004), 283-291.

[Barabasi and Albert 99] A. Barabasi and R. Albert. "Emergence of Scaling in Random Networks." Science 286 (1999), 509-512.

[Bassetti et al. 07] F. Bassetti, M. Cosentino Lagomarsino, B. Bassetti, and P. Jona. "Random Networks Tossing Biased Coins." Phys. Rev. E 75 (2007), 056109.

[Bollobás 01] B. Bollobás. Random Graphs, second edition,Cambridge Studies in Advanced Mathematics 73. Cambridge, UK: Cambridge University Press, 2001.

[Bollobás and Riordan 03a] B. Bollobás and O. M. Riordan. "Mathematical Results on Scale-Free Random Graphs." In Handbook of Graphs and Networks, edited by S. Bornholdt and H. G. Schuster, pp. 1-34. Weinheim: Wiley-VCH, 2003.

[Bollobás and Riordan 03b] B. Bollobás and O. Riordan. "Robustness and Vulnerability of Scale-Free Random Graphs." Internet Math. 1:1 (2003), 1-35.

[Bollobás and Riordan 04a] B. Bollobás and O. Riordan. "Coupling Scale-Free and Classical Random Graphs." Internet Math. 1:2 (2004), 215-225.

[Bollobás and Riordan 04b] B. Bollobás and O. Riordan. "The Diameter of a ScaleFree Random Graph." Combinatorica 24:1 (2004), 5-34.

[Bollobás et al. 03] B. Bollobás, C. Borgs, J. Chayes, and O. Riordan. "Directed ScaleFree Graphs." In Proceedings of the Fourteenth Annual ACM-SIAM Symposium on Discrete Algorithms, pp. 132-139. Philadelphia: SIAM, 2003.

[Chen et al. 05] Y. Chen, P. Diaconis, S. P. Holmes, and J. S. Liu. "Sequential Monte Carlo Methods for Statistical Analysis of Tables." J. Amer. Statist. Assoc. 100:469 (2005), 109-120.

[Chung and Lu 03] F. Chung and L. Lu. "The Average Distance in a Random Graph with Given Expected Degrees." Internet Math. 1:1 (2003), 91-113. 
[Chung and Lu 04] F. Chung and L. Lu. "The Small World Phenomenon in Hybrid Power Law Graphs." In Complex Networks, Lecture Notes in Physics 650, pp. 89104. Berlin: Springer, 2004.

[Chung and Lu 06a] F. Chung and L. Lu. Complex Graphs and Networks, CBMS Regional Conference Series in Mathematics 107. Washington, DC: American Mathematical Society, 2006.

[Chung and Lu 06b] F. Chung and L. Lu. "The Volume of the Giant Component of a Random Graph with Given Expected Degrees." SIAM J. Discrete Math. 20:2 (2006), 395-411.

[Cosentino Lagomarsino et al. 05] M. Cosentino Lagomarsino, B. Bassetti, and P. Jona. "The Large-Scale Logico-chemical Structure of a Transcriptional Regulation Network." Preprint, 2005. ArXiv:q-bio/0502017v1.

[Durrett 07] R. Durrett. Random Graph Dynamics, Cambridge Series in Statistical and Probabilistic Mathematics. Cambridge, UK: Cambridge University Press, 2007.

[Ellis 06] R. S. Ellis. The Theory of Large Deviation and Applications to Statistical Mechanics. Lectures for the International Seminar on Extreme Events in Complex Dynamics. Dresden: Max-Planck-Institut, 2006.

[Erdős and Rényi 64] P. Erdős and A. Rényi. "On Random Matrices." Magyar Tud. Akad. Mat. Kutató Int. Közl, 8 (1964), 455-461.

[Erdős and Rényi 68] P. Erdős and A. Rényi. "On Random Matrices. II." Studia Sci. Math. Hungar. 3 (1968), 459-464.

[Feller 48] W. Feller. "On Probability Problems in the Theory of Counters." In Studies and Essays Presented to R. Courant on His 60th Birthday, January 8, 1948, edited by K. O. Freidrichs, pp. 105-115. New York: Interscience Publishers, Inc., 1948.

[Feller 71] W. Feller. An Introduction to Probability Theory and Its Applications, Vol. II, second edition. New York: John Wiley \& Sons Inc., 1971.

[Fortini et al. 96] S. Fortini, L. Ladelli, and E. Regazzini. "A Central Limit Problem for Partially Exchangeable Random Variables." Teor. Veroyatnost. i Primenen. 41:2 (1996), 353-379.

[Galambos 87] J. Galambos. The Asymptotic Theory of Extreme Order Statistics, second edition. Melbourne, FL: Robert E. Krieger Publishing Co. Inc., 1987.

[Gradshteyn and Ryzhik 00] I. S. Gradshteyn and I. M. Ryzhik. Table of Integrals, Series, and Products, sixth edition. New York: Academic Press Inc., 2000.

[Grandell 97] J. Grandell. Mixed Poisson Processes, Monographs on Statistics and Applied Probability 77. London: Chapman \& Hall, 1997.

[Guelzim et al. 02] N. Guelzim, S. Bottani, P. Bourgine, and K. Kepes. "Topological and Causal Structure of the Yeast Transcriptional Regulatory Network." Nat. Genet. 31 (2002), 60-63.

[Harbison et al. 04] C. Harbison, D. B. Gordon, T. I. Lee, N. J. Rinaldi, K. D. Macisacc, T. W. Danford, N. M. Hannett et al. "Transcriptional Regulatory Code of a Eukaryotic Genome." Nature 431 (2004), 99-104. 
[Itzkovitz et al. 03] S. Itzkovitz, R. Milo, N. Kashtan, G. Ziv, and U. Alon. "Subgraphs in Random Networks." Phys. Rev. E 68 (2003), 026127.

[Kallenberg 02] O. Kallenberg. Foundations of Modern Probability. Probability and Its Applications, second edition. New York: Springer-Verlag, 2002.

[Kolchin 99] V. F. Kolchin. Random Graphs, Encyclopedia of Mathematics and Its Applications, 53. Cambridge, UK: Cambridge University Press, 1999.

[Lee et al. 02] T. Lee N. Rinaldi, F. Robert, D. T. Odorn, Z. Bar-Joseph, G. K. Gerber, N. M. Hannett et al. "Transcriptional Regulatory Networks in Saccharomyces cerevisiae." Science 298 (2002), 799.

[Levitskaya 05] A. A. Levitskaya. "Systems of Random Equations over Finite Algebraic Structures." Kibernet. Sistem. Anal. 41:1 (2005), 82-116, 190.

[MacKay 99] D. J. C. MacKay. "Good Error-Correcting Codes Based on Very Sparse Matrices." IEEE Transactions on Information Theory 45 (1999), 399-431.

[Magnus et al. 66] W. Magnus, F. Oberhettinger, and R. P. Soni. Formulas and Theorems for the Special Functions of Mathematical Physics, third edition. New York: Springer-Verlag, 1966.

[Matias 06] S. Matias, C. amd Schbath, E. Birmela, J. J. Daudin, and S. Robin. "Networks Motifs: Mean and Variance for the Count." REVSTAT 4:1 (2006), 31-51.

[Mézard et al. 02] M. Mézard, G. Parisi, and R. Zecchina. "Analytic and Algorithmic Solution of Random Satisfiability Problems." Science 297 (2002), 812-815.

[Mézard et al. 03] M. Mézard, F. Ricci-Tersenghi, and R. Zecchina. "Alternative Solutions to Diluted $p$-spin Models and XORSAT Problems." J. Stat. Phys. 111 (2003), 505-533.

[Milo et al. 02] R. Milo, S. Shen-Orr, S. Itzkovitz, N. Kashtan, D. Chklovskii, and U. Alon. "Network Motifs: Simple Building Blocks of Complex Networks." Science 298 (2002), 824-827.

[Milo et al. 04] R. Milo, S. Itzkovitz, N. Kashtan, R. Levitt, S. Shen-Orr, I. Ayzenshtat, M. Sheffer, and U. Alon. "Superfamilies of Evolved and Designed Networks." Science 303 (2004), 1538-1542.

[Montoya et al. 06] J. M. Montoya, S. L. Pimm, and R. V. Sole. "Abstract Ecological Networks and Their Fragility." Nature 442 (2006), 259-264.

[Murayama and Okada 03] T. Murayama and M. Okada. "One Step RSB Scheme for the Rate Distortion Function." J. Phys. 36 (2003), 11123-11130.

[Newman 03a] M. E. J. Newman. "Random Graphs as Models of Networks." In Handbook of Graphs and Networks, edited by S. Bornholdt and H. G. Schuster, pp. 3568. Weinheim: Wiley-VCH, 2003.

[Newman 03b] M. E. J. Newman. "The Structure and Function of Complex Networks." SIAM Rev. 45:2 (2003), 167-256.

[Newman et al. 06] M. E. J. Newman, A. L. Barabasi, and D. J. Watts. The Structure and Dynamics of Networks. Princeton, NJ: Princeton University Press, 2006.

[Prudnikov et al. 86] A. P. Prudnikov, Yu. A. Brychkov, and O. I. Marichev. Integrals and Series, Vol. 1. New York: Gordon \& Breach Science Publishers, 1986. 
[Puri and Goldie 79] P. S. Puri and C. M. Goldie. "Poisson Mixtures and Quasi-infinite Divisibility of Distributions." J. Appl. Probab. 16:1 (1979), 138-153.

[Rao et al. 96] A. Rao, R. Jana, and S. Bandyopadhyay. "A Markov Chain Monte Carlo Method for Generating Random (0,1)-matrices with Given Marginals." Sankhyā 58:A (1996), 225-242.

[Regazzini and Sazonov 97] E. Regazzini and V. V. Sazonov. "On the Central Limit Problem for Partially Exchangeable Random Variables with Values in a Hilbert Space." Teor. Veroyatnost. i Primenen. 42:4 (1997), 796-812.

[Salgado et al. 01] H. Salgado, A. Santos-Zavaleta, S. Gamma-Castro, D. MillánZárate, E. Díaz-Peredo, F. Sánchez-Solano, E. Pŕez-Rueda, C. BonavidesMartínez, and J. Collado-Vides. "RegulonDB (Version 3.2): Transcriptional Regulation and Operon Organization in Escherichia coli K-12." Nucleic Acids Res. 29 (2001), 72-74.

[Shen-Orr et al. 02] S. Shen-Orr, R. Milo, S. Mangan, and U. Alon. "Network Motifs in the Transcriptional Regulation Network of Escherichia coli." Nat. Genet. 31 (2002), 64-68.

[Steutel and van Harn 04] F. W. Steutel and K. van Harn. Infinite Divisibility of Probability Distributions on the Real Line, Pure and Applied Mathematics 259. New York: Marcel Dekker Inc., 2004.

[Strogatz 01] S. H. Strogatz. "Exploring Complex Networks." Nature 410 (2001), 268276.

[Watts and Strogatz 98] D. J. Watts and S. H. Strogatz. "Collective Dynamics of 'Small-World' Networks." Nature 393 (1998), 440-442.

[Willmot 90] G. E. Willmot. "Asymptotic Tail Behaviour of Poisson Mixtures with Applications." Adv. in Appl. Probab. 22:1 (1990), 147-159.

[Wolf and Arkin 03] D. Wolf and A. Arkin. "Motifs, Modules and Games in Bacteria." Curr. Opin. Microbiol. 6 (2003), 125.

[Yeger-Lotem et al. 04] E. Yeger-Lotem, S. Sattath, N. Kashtan, S. Itzkovitz, R. Milo, R. Y. Pinter, U. Alon, and H. Margalit. "Network Motifs in Integrated Cellular Networks of TranscriptionRegulation and RroteinProtein Interaction." Proc. Natl. Acad. Sci. 101:16 (2004), 5934-5939.

F. Bassetti, Università degli Studi di Pavia, Dip. Matematica, Via Ferrata 1, 27100 Pavia, Italy (federico.bassetti@unipv.it)

M. Cosentino Lagomarsino, Università degli Studi di Milano, Dip. Fisica and I.N.F.N., Via Celoria 16, 20133 Milano, Italy (marco.cosentino-lagomarsino@unimi.it)

S. Mandrà, Università degli Studi di Milano, Dip. Fisica and I.N.F.N., Via Celoria 16, 20133 Milano, Italy (salvatore.mandra@mi.infn.it)

Received July 24, 2007; accepted April 3, 2008. 